\title{
RESONANCES OF ENCLOSED SHALLOW WATER BASINS WITH SLENDER FLOATING ELASTIC BODIES
}

\author{
by T.K. Papathanasiou $\left({ }^{\mathrm{a}^{*}}\right)$ and K.A. Belibassakis $\left({ }^{b}\right)$ \\ ${ }^{a}$ Department of Civil \& Environmental Engineering, Brunel University London, \\ Uxbridge UB8 3PH, UK \\ ${ }^{\mathrm{b}}$ School of Naval Architecture and Marine Engineering, National Technical \\ University of Athens, Zografos 15773, Athens, Greece \\ $\left(^{*}\right)$ Corresponding author: email: theodosios.papathanasiou@brunel.ac.uk
}

\begin{abstract}
A model for the hydroelastic response of enclosed shallow water basins with floating slender plates of large span, in variable bathymetry is presented. The eigenproblem for the system's resonant behaviour is formulated in the strong and variational form. Based on the variational form, and special hydroelastic finite elements, a quadratic eigenvalue problem is derived for the eigenfrequencies and eigenmodes of the basinfloating body system. Benchmark examples, for simplified configurations, are used to study the effects of parameters like the plate's span, position, draft, mass, stiffness and depth of the basin. It is observed that the presence of the plate can alter significantly the eigenstates of the pond if the depth of the basin is relatively small, compared to the plate draft. In such cases the plate span and position play a crucial role. The hydroelastic analysis can be important even in cases where the depth increases and the upper surface elevation differences, in the presence or absence of the floating plate, appear to be less significant. This is due to the importance in accurately calculating the curvature and hence bending moments inside the plate. The proposed model could be relevant to the seiche formation analysis in reservoirs with floating photovoltaic platforms or ice-covered lakes.
\end{abstract}

Keywords: VLFS hydroelasticity; seiches; shallow water basins; floating photovoltaic platforms; finite elements

\section{Introduction}

The formation of standing waves in enclosed or partially enclosed bodies of water, such as lakes, bays, reservoirs and harbours has been documented in several occasions $[1,2,3]$. The term seiche has been popularised by the Swiss hydrologist FrançoisAlphonse Forel for these rhythmic oscillations, initially in the context of limnology [4]. Seiches fall in the category of long period (infragravity) waves and are often 
imperceptible to the naked eye due to their very long wavelengths [5]. In many cases the free water surface elevation or depression, associated with the formation of a seiche, is only a few centimetres. However, extreme seiches, associated to wave amplitudes of several meters, have been documented, e.g. in Lake Erie [6].

The formation of seiches is attributed principally to atmospheric pressure changes or ground excitation, for example due to earthquakes [7, 8]. An impressive event regarding the formation of seiches in Scottish lakes Loch Long, Loch Lomond, Loch Katrine and Loch Ness, due to an earthquake that hit Lisbon Portugal (epicentre about $200 \mathrm{~km}$ west-southwest of Cape St. Vincent) was documented in 1755 [9]. More recently, seiches with amplitude of approximately 2 meters were observed in the Norwegian fjord Sognefjorden, during the 2011 Tohoku earthquake [10]. Seiches in smaller basins (e.g. reservoirs) have also been attributed to earthquakes [2].

The case of seiche type standing waves in ice-covered lakes is a related subject that has been investigated by several authors $[6,11]$. The long waves that appear in such cases might have altered characteristics due to the interaction with floating ice formations. If floating ice formations have large horizontal dimensions, with respect to their thickness, hydroelastic effects might become significant and influence the response of the lake-slender floating body system. In such cases, large areas of hydroelastic interactions are bound to manifest and it can be conjectured that the response of the system will be altered. I. V. Sturova [12] presented a model for the analysis and simulation of seiches in lakes with large slender floating bodies, representing floating ice formations. This model referred to a fully covered basin with either freely floating ice or fixed at the basin boundaries. The outcome of this study was that the presence of freely floating, slender structures of large span, does not affect significantly the resonant behaviour of the lake during a seiche. In particular, very slight differences between the eigenfrequencies of the lake with free surface and the same lake with large floating ice formations were reported. The situation was different in the case of ice formations that were rigidly fixed at the lake shore and extending as floating cantilevers. In such cases, the effect of the ice cover was found to be significant in the system response, particularly near the basin boundaries.

A recent concept related to energy production is that of floating photovoltaic systems (floating PV) [13, 14]. Such installations might also be relevant to the phenomenon of hydroelastic resonances. Large floating photovoltaic platforms are placed into lakes or water reservoirs, similar to the one shown in Fig 1. (left), for the exploitation of solar energy but also for preventing excessive water evaporation. It has been established that the efficiency of a photovoltaic panel increases when they are floating on water, since the latter acts as a coolant and the PV system operates at a lower temperature [13]. A floating PV facility of very large horizontal dimensions (approximately 57000 $\mathrm{m}^{2}$ ) is currently installed in Queen Elizabeth II pond in Surrey [14]. The horizontal span of such a platform classifies it in the category of very large floating structures. The distinguishing features regarding the analysis of floating PV platforms in ponds are that the pond area might be not much larger than that of the platform, while the 
maximum depth is not necessarily much larger than the platform draft. In such cases, the overlap area and the platform draft, along with the macroscopic stiffness of the platform could be crucial parameters affecting the hydroelastic system response. Hydroelastic models could thus be a valuable tool in assessing the structural integrity of this kind of multi-million pound installations.
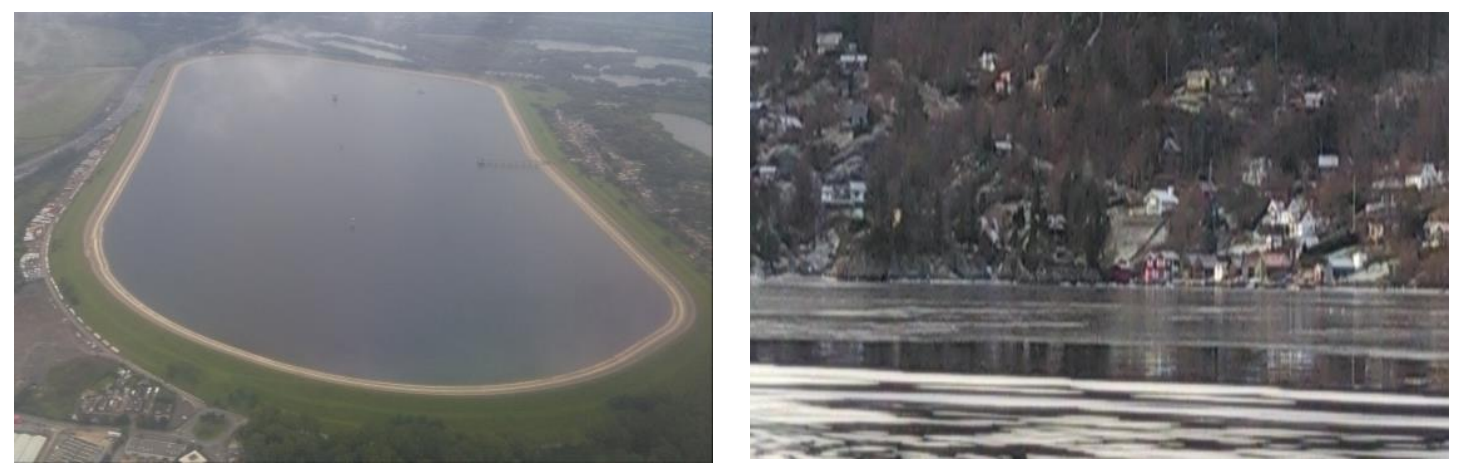

Figure 1. Left: enclosed water reservoir (West London area, UK), Right: partially enclosed and ice-covered basin in a Norwegian fjord (near Stavanger, Norway).

Finally, it should be noted that resonant hydroelastic oscillations are also observed during the interaction of ice-shelves with oceanic waves. Several authors have studied this type of hydroelastic resonant behaviour $[15,16,17]$ while different models and solution methodologies have been presented to this end [18-21].

The aim of the present study is to assess the following possibility: 'will the presence of freely floating Very Large Floating Structure (VLFS) shift the natural frequencies of a pond and in general alter the response of the confined shallow basin with respect to seiche formation?' Applications could include the structural integrity assessment of large floating PV installations or ice covered basins, like the fjord region shown in figure 1 (right). The study is organised as follows: sections 2 and 3 present the geometry and governing equations for the eigenproblem considered. In section 3, the variational form of the eigenproblem is derived and its properties are analysed in section 4. Subsequently, in order to study the effects of the system parameters (e.g. plate span, depth, plate material properties) a shallow basin of infinite width is considered. In section 5, a hydroelastic finite element procedure for the determination of eigenfrequencies and eigenmodes of such a basin is briefly discussed. The rest of the paper includes numerical examples and parametric studies on the basin-floating structure response. In particular, the effects of the plate's span, position, draft, mass, stiffness and the basin depth are studied. Cases of multiple floating plates and variable bathymetry basins are included. The basic findings and conclusions are finally summarised and discussed along with planned future developments and extensions of the proposed model. 


\section{The Hydroelastic Model}

\subsection{Domain Geometry}

Let $\Omega \subset \mathbb{R}^{2}$ be closed and bounded with smooth boundary $\Gamma$. For $N \in \mathbb{N}$, the open subsets $\Omega_{j}, j=1,2, \ldots, N$ of $\Omega$ with smooth boundaries $\Gamma_{j}$ are introduced, such that $\Omega_{j} \subset \Omega$ and $\bar{\Omega}_{i} \cap \bar{\Omega}_{j}=\varnothing$ for $i \neq j$ and $i, j=1,2, \ldots, N$, where $\bar{\Omega}_{j}=\Omega_{j} \cup \Gamma_{j}$. The configuration is depicted in Fig. 2. Finally, we denote

$$
\begin{gathered}
\Omega_{0}=\Omega-\bigcup_{j=1}^{N} \bar{\Omega}_{j} \text { and } \\
\Gamma_{0}=\Gamma \cup \Gamma_{1} \cup \Gamma_{2} \cup \ldots \cup \Gamma_{N} .
\end{gathered}
$$

The geometry introduced is aimed to represent a confined bulk of water, like a lake or a pond, with multiple very large bodies of shallow draft floating freely on the water surface. The areas occupied by the floating bodies are disjoint. Furthermore the bathymetry function $b(x, y): \Omega \rightarrow \mathbb{R}_{+}$and the positive constants $\rho, \rho_{j}$, with $\rho_{j}<\rho$, denoting the density of water and the density of each plate respectively, are introduced. According to Archimedes principle, the draft of each plate is $d_{j}=\rho_{j} \tau_{j} / \rho$, where $\tau_{j}$ is the thickness distribution of floating body $j$. The domain occupied by the fluid is thus defined as

$$
D_{0}=\left\{(x, y, z) \in \mathbb{R}^{3} \mid z \in\left[-b,-d_{j}\right],(x, y) \in \Omega_{j}, j=0,1,2, \ldots, N\right\},
$$

while each floating body occupies one of the domains

$$
D_{j}=\left\{(x, y, z) \in \mathbb{R}^{3} \mid z \in\left[-d_{j}, \tau_{j}-d_{j}\right],(x, y) \in \Omega_{j}, j=1,2, \ldots, N\right\},
$$

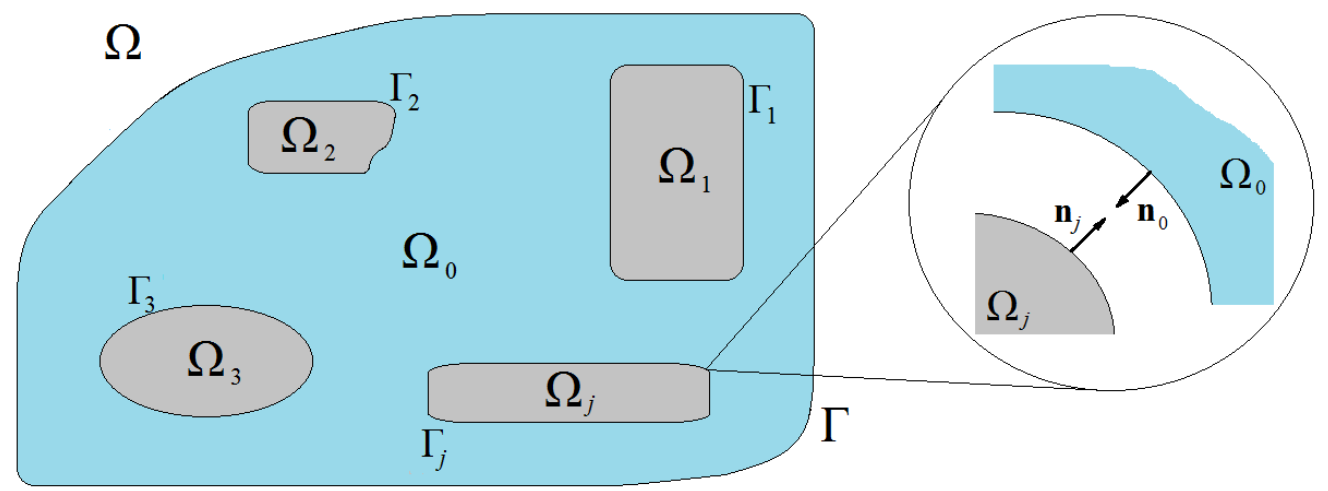

Figure 2. Multiple Very Large Floating Bodies of shallow draft in a confined, shallow water environment. 


\subsection{Governing Equations}

In order to derive a relatively simple but realistic model of hydroelastic interactions regarding multiple floating bodies in an enclosed shallow water basin, the following set of assumptions will be adopted.

(A1) The fluid motion is irrotational and governed by linear shallow water hydrodynamics. This assumption is compatible with the analysis of standing waves with large wavelength in lakes or ponds, when the depth is much smaller than the considered wavelengths.

(A2) The floating bodies are elastic, slender and subject to the Kirchhoff-Love assumptions for bending of thin plates. In particular it is $\tau_{j} / \operatorname{diam}\left(\Omega_{j}\right)<<1$, for $j=1,2, \ldots, N$. The plate material is an isotropic solid satisfying Hooke's law. Its elastic modulus is $E_{j}>0$ and its Poisson's ratio $v_{j} \in(-1,0.5]$.

(A3) The floating bodies do not drift in the horizontal plane $x-y$ and only deflect along the $z$-axis.

Effects of Coriolis force are not considered in this study. Their effect has been found by I. V. Sturova [12] to be negligible in the hydroelastic response of ice covered lakes. It is natural to assume that the same will hold for water reservoirs that have much smaller span. The irrotationality and shallow water conditions in assumption (A1) allow for the introduction of the scalar functions $\Phi_{j}: \Omega_{j} \times(0, T] \rightarrow \mathbb{R}$, $j=0,1,2, \ldots, N$, representing the fluid velocity potential. The velocity vector, with velocity components along the $x$ and $y$ direction, in each region is then defined as

$$
\mathbf{u}_{j}=\nabla \Phi_{j},(x, y) \in \Omega_{j} .
$$

Given shallow water conditions, while considering waves of small amplitude, the velocity potential $\Phi_{0}$, in the region where no floating plates exist, satisfies the wave equation

$$
\partial_{t t} \Phi_{0}-g \nabla \cdot\left(b \nabla \Phi_{0}\right)=0, \text { in } \Omega_{0} \times(0, T],
$$

where $\partial_{t}$ denotes differentiation with respect to time and $g$ is the acceleration of gravity. The free surface elevation in $\Omega_{0}$ is [22]

$$
\eta_{0}=-g^{-1} \partial_{t} \Phi_{0}
$$

Denoting the fluid upper surface elevation (plate deflection) in $\Omega_{j}$ as $\eta_{j}: \Omega_{j} \times(0, T] \rightarrow \mathbb{R}$, continuity of fluid mass underneath the floating plate, leads to the equation 


$$
\partial_{t} \eta+\nabla \cdot\left[\left(b-d_{j}\right) \nabla \Phi_{j}\right]=0, \text { in } \Omega_{j} \times(0, T]
$$

Conservation of mass and energy at the interfaces $\Gamma_{j}$ between $\Omega_{j}, j=1,2, \ldots, N$ and $\Omega_{0}$ imply the interface conditions [22]

$$
\left(b-d_{j}\right) \nabla \Phi_{j} \cdot \mathbf{n}_{j}=-b \nabla \Phi_{0} \cdot \mathbf{n}_{0} \text { and } \partial_{t} \Phi_{j}=\partial_{t} \Phi_{0} \text { on } \Gamma_{j} \times(0, T]
$$

where $\mathbf{n}_{j}, \mathbf{n}_{0}$ denote the outward unit normal on $\Omega_{j}$ and $\Omega_{0}$ respectively.

Finally, rigid wall conditions, yielding full reflection, apply on the pond perimeter $\Gamma$, where consequently the fluid velocity is zero and thus

$$
\nabla \Phi_{0} \cdot \mathbf{n}_{0}=0, \text { on } \Gamma \times(0, T]
$$

In order to formulate the hydroelastic interaction model, equations (2.6) and (2.8) must be supplemented by the plate deflection model. Assuming that the floating plates are always in contact with the fluid, the upper surface elevation of the fluid in $\Omega_{j}$ coincides with the floating plate deflection. Thus, the pressure on the upper surface of the fluid acts as a distributed load on the plate. Based on assumptions (A2) and (A3), the equation governing the response of floating plate $j$ is

$$
\rho_{j} \tau_{j} \partial_{t t} \eta_{j}+\Delta\left(k_{j} \Delta \eta_{j}\right)+\rho g \eta_{j}+\rho \partial_{t} \Phi_{j}=0
$$

where $k_{j}=\frac{E_{j} \tau_{j}^{3}}{12\left(1-v_{j}^{2}\right)}$.

The plate bending moment tensor for floating plate $j$, in vector form, is

$$
\mathbf{M}_{j}\left(\eta_{j}\right)=k_{j}\left[\begin{array}{ccc}
1 & v_{j} & 0 \\
v_{j} & 1 & 0 \\
0 & 0 & 1-v_{j}
\end{array}\right]\left[\begin{array}{l}
\partial_{x x} \eta_{j} \\
\partial_{y y} \eta_{j} \\
\partial_{x y} \eta_{j}
\end{array}\right]
$$

Freely floating boundary conditions are realised by imposing the normal bending moment and active shear force to be zero at the lateral boundary of each plate [23], that is

$$
\mathbf{M}_{n}\left(\eta_{j}\right)=\mathbf{n}_{j}^{T} \mathbf{M}\left(\eta_{j}\right) \mathbf{n}_{j}=0 \text { and } \mathbf{Q}\left(\eta_{j}\right)+\nabla \mathbf{T}\left(\eta_{j}\right) \cdot \mathbf{t}_{j}=0, \text { on } \Gamma_{j}, j=1,2, \ldots, N
$$

where $\mathbf{T}\left(\eta_{j}\right)=\mathbf{n}_{j}^{T} \mathbf{M}\left(\eta_{j}\right) \mathbf{t}_{j}$ is the twisting moment and $\mathbf{Q}\left(\eta_{j}\right)=\nabla \cdot \mathbf{M}\left(\eta_{j}\right) \mathbf{n}_{j}$ is the shear force, with $\mathbf{t}_{j}$ being the unit tangent vector on $\Gamma_{j}$.

The strong form of the Initial Boundary Value (IBV) hydroelastic problem in the enclosed shallow basin is to find $\Phi_{j}: \Omega_{j} \times(0, T] \rightarrow \mathbb{R}, \quad j=0,1,2, \ldots, N$ and 
$\eta_{j}: \Omega_{j} \times(0, T] \rightarrow \mathbb{R}$ for $j=1,2, \ldots, N$ that satisfy the field equations (2.6), (2.8) and (2.11), interface conditions (2.9) and boundary conditions (2.10), (2.13), along with appropriate initial conditions. In the following section, after introducing convenient nondimensional variables, the weak form of the respective hydroelastic problem will be derived in the frequency domain, leading to a quadratic eigenvalue problem in variational form.

\section{The Hydroelastic Eigenproblem}

Introducing the characteristic length $l_{P}=\max _{j \in[1, N] \cap \mathbb{N}}\left\{\operatorname{diam}\left(\Omega_{j}\right)\right\}$, the following nondimensional quantities are defined

$$
(\tilde{x}, \tilde{y})=(x, y) / l_{P}, \tilde{t}=t\left(g / l_{P}\right)^{1 / 2} \tilde{\eta}_{j}=\tilde{\eta}_{j} / l_{P} \text { and } \tilde{\Phi}_{j}=\Phi_{j} g^{-1 / 2} l_{P}^{-3 / 2} \text {. }
$$

Introducing standing wave solutions of the form $\eta_{j}(x, y, t)=\operatorname{Re}\left\{\eta_{j}(x, y) e^{i \omega t}\right\}$, $j=1,2, \ldots, N$, and $\Phi_{j}(x, y, t)=\operatorname{Re}\left\{\Phi_{j}(x, y) e^{i \omega t}\right\}, \quad j=0,1,2, \ldots, N$ and using the nondimensional quantities defined in (3.1) governing equations, in steady state conditions and nondimensional form, become (after dropping tildes)

$$
\begin{gathered}
\Delta\left(K_{j} \Delta \eta_{j}\right)+\left(1-\omega^{2} m_{j}\right) \eta_{j}+i \omega \Phi_{j}=0 \text { in } \Omega_{j} \text { for } j=1,2, \ldots, N, \\
\nabla \cdot\left(B_{j} \nabla \Phi_{j}\right)+i \omega \eta_{j}=0, \text { in } \Omega_{j} \text { for } j=1,2, \ldots, N \text { and } \\
\nabla \cdot\left(B_{0} \nabla \Phi_{0}\right)+\omega^{2} \Phi_{0}=0, \text { in } \Omega_{0},
\end{gathered}
$$

where $m_{j}=l_{P}^{-1} d_{j}, K_{j}=\left(\rho g l_{P}^{4}\right)^{-1} k_{j}, B_{j}=l_{P}^{-1} b-m_{j}$ for $j=1,2, \ldots, N$ and $B_{0}=b / l_{P}$.

The above equations are supplemented by the interface conditions

$$
B_{j} \nabla \Phi_{j} \cdot \mathbf{n}_{j}=-B_{0} \nabla \Phi_{0} \cdot \mathbf{n}_{0} \text { and } \Phi_{j}=\Phi_{0} \text { on } \Gamma_{j}, j=1,2, \ldots, N,
$$

the zero normal bending moment and active shear conditions at the lateral boundary of the floating plates, that is Eqs. (2.13) and the zero normal velocity condition on $\Gamma$.

The variational form of the above stated problem can be derived by multiplying equations (3.2), (3.3) and (3.4) by the conjugate of suitable weight functions $\chi_{j},-w_{j}$, $j=1,2, \ldots, N$ and $-w_{0}$ respectively, integration over the appropriate domain and application of the Green-Gauss theorem. In the case of the fourth order term $\Delta\left(K_{j} \Delta \eta_{j}\right)$, two repeated applications of the Green-Gauss theorem and appropriate handling of the resulting boundary integrals, using the surface and normal gradient operators [24], the surface divergence theorem and Stokes theorem, results to 


$$
\begin{aligned}
\int_{\Omega_{j}} \bar{\chi}_{j} \Delta( & \left.K_{j} \Delta \eta_{j}\right) d x d y=\int_{\Omega_{j}} K_{j} v_{j} \Delta \bar{\chi}_{j} \Delta \eta_{j} d x d y \\
& +\int_{\Omega_{j}} K_{j}\left(1-v_{j}\right)\left(\partial_{x x} \bar{\chi}_{j} \partial_{x x} \eta_{j}+\partial_{y y} \bar{\chi}_{j} \partial_{y y} \eta_{j}+2 \partial_{x y} \bar{\chi}_{j} \partial_{x y} \eta_{j}\right) d x d y \\
& +\int_{\Gamma_{j}} \mathbf{M}_{n}\left(\eta_{j}\right) \nabla \bar{\chi}_{j} \cdot \mathbf{n}_{j} d s-\int_{\Gamma_{j}}\left(\mathbf{Q}\left(\eta_{j}\right)+\nabla \mathbf{T}\left(\eta_{j}\right) \cdot \mathbf{t}_{j}\right) \bar{\chi}_{j} d s
\end{aligned}
$$

Finally, the weak form for the hydroelastic problem, taking also into account (2.13), is

$$
\begin{gathered}
a_{j}\left(\bar{\chi}_{j}, \eta_{j}\right)+\int_{\Omega_{j}} \bar{\chi}_{j} \eta_{j} d x d y+i \omega \int_{\Omega_{j}} \bar{\chi}_{j} \Phi_{j} d x d y-\omega^{2} \int_{\Omega_{j}} m \bar{\chi}_{j} \eta_{j} d x d y=0, \\
\int_{\Omega_{j}} B_{j} \nabla \bar{w}_{j} \cdot \nabla \Phi_{j} d x d y-i \omega \int_{\Omega_{j}} \bar{w}_{j} \eta_{j} d x d y-\int_{\Gamma_{j}} \bar{w}_{j} B_{j} \nabla \Phi_{j} \cdot \mathbf{n}_{j} d s=0 \text { and } \\
\int_{\Omega_{0}} B_{0} \nabla \bar{w}_{0} \cdot \nabla \Phi_{0} d x d y-\omega^{2} \int_{\Omega_{0}} \bar{w}_{0} \Phi_{0} d x d y-\int_{\Gamma_{j}} \bar{w}_{0} B_{0} \nabla \Phi_{0} \cdot \mathbf{n}_{0} d s=0,
\end{gathered}
$$

where,

$$
\begin{aligned}
a_{j}\left(\bar{\chi}_{j}, \eta_{j}\right) & =\int_{\Omega_{j}} K_{j} v_{j} \Delta \bar{\chi}_{j} \Delta \eta_{j} d x d y \\
& +\int_{\Omega_{j}} K_{j}\left(1-v_{j}\right)\left(\partial_{x x} \bar{\chi}_{j} \partial_{x x} \eta_{j}+\partial_{y y} \bar{\chi}_{j} \partial_{y y} \eta_{j}+2 \partial_{x y} \bar{\chi}_{j} \partial_{x y} \eta_{j}\right) d x d y
\end{aligned}
$$

and the homogeneous Neumann condition for $\Phi_{0}$ on $\Gamma$, i.e. $\int_{\Gamma} \bar{w}_{0} B_{0} \nabla \Phi_{0} \cdot \mathbf{n}_{0} d s=0$, has been implemented. Assuming further that the weight functions $\bar{w}_{j}$ and $\bar{w}_{0}$, satisfy the interface condition $i \omega w_{j}=i \omega w_{0}$ on $\Gamma_{j}$, for $j=1,2, \ldots, N$ and taking into account interface condition (2.9a) and the fact that $\mathbf{n}_{j}=-\mathbf{n}_{0}$ (see also Fig. 2), it is

$$
\int_{\Gamma_{j}}\left(\bar{w}_{j} B_{j} \nabla \Phi_{j} \cdot \mathbf{n}_{j}+\bar{w}_{0} B_{0} \nabla \Phi_{0} \cdot \mathbf{n}_{0}\right) d s=0
$$

The variational form of the eigenvalue problem results by the summation of (3.7), (3.8) and (3.9) over all $j$ and can be stated as:

find $\omega \in \mathbb{C}, \eta_{j} \in H^{2}\left(\Omega_{j} ; \mathbb{C}\right), \Phi_{j} \in H^{1}\left(\Omega_{j} ; \mathbb{C}\right) \quad j=1,2, \ldots, N$ and $\Phi_{0} \in H^{1}\left(\Omega_{0} ; \mathbb{C}\right)$, such that for all admissible $\bar{\chi}_{j} \in H^{2}\left(\Omega_{j} ; \mathbb{C}\right),-\bar{w}_{j} \in H^{1}\left(\Omega_{j} ; \mathbb{C}\right) \quad j=1,2, \ldots, N$ and $-\bar{w}_{0} \in H^{1}\left(\Omega_{0} ; \mathbb{C}\right)$, it is

$$
\begin{aligned}
& \sum_{j=1}^{N}\left[a_{j}\left(\bar{\chi}_{j}, \eta_{j}\right)+\int_{\Omega_{j}} \bar{\chi}_{j} \eta_{j} d x d y+\int_{\Omega_{j}} B_{j} \nabla \bar{w}_{j} \cdot \nabla \Phi_{j} d x d y-\omega^{2} \int_{\Omega_{j}} m_{j} \bar{\chi}_{j} \eta_{j} d x d y\right] \\
& +i \omega \sum_{j=1}^{N}\left[\int_{\Omega_{j}}\left(\bar{\chi}_{j} \Phi_{j}-\bar{w}_{j} \eta_{j}\right) d x d y\right]+\int_{\Omega_{0}} B_{0} \nabla \bar{w}_{0} \cdot \nabla \Phi_{0} d x d y-\omega^{2} \int_{\Omega_{0}} \bar{w}_{0} \Phi_{0} d x d y=0
\end{aligned}
$$


where $H^{k}\left(\Omega_{j} ; \mathbb{C}\right)$ is the space of complex-valued functions $f$ defined on $\Omega_{j}$ and have Lebesgue-square integrable derivatives $\frac{\partial^{a_{1}+a_{2}} f}{\partial x^{a_{1}} \partial y^{a_{2}}}$ for all non-negative integers $a_{1}+a_{2} \leq k \in \mathbb{N}_{0}$. It is expected that the solution behaves as non-propagating waves and thus $\nabla\left[\arg \left(\Phi_{j}\right)\right]=\nabla\left[\arg \left(\eta_{j}\right)\right]=0$.

\section{Properties of the Eigenstates}

\subsection{Mass Conservation and Mean Value of the Wave Fields}

In this section the mass conservation properties of the system will be examined. It will be demonstrated, using the variational form, that mass conservation enables the estimation of the wave fields $\eta_{j}$ and $\Phi_{j}$ in the mean value sense. Since the shallow water environment under consideration is confined by rigid boundaries with no inflow or outflow conditions, and no mass sources or sinks are present, physical intuition suggests that the total amount of mass should remain constant. Consequently, the mean value of the upper surface elevation, that being the plate deflection in the hydroelastic interaction regions, or free surface water waves in the unoccupied portion of the pond, should equal that of the undisturbed state, which is zero. The following proposition summarizes these results.

PROPOSITION 1 Let $|\Omega|$ denote the measure of $\Omega$ and set $m_{0}=0$. At each eigenstate $n \in \mathbb{N}$

(i) the mean value of the upper surface elevation in $\Omega$ is zero and

(ii) the mean value of the velocity potential in $\Omega$ is proportional to the mean value of $m \eta$, i.e

$$
\begin{gathered}
|\Omega|^{-1} \sum_{j=0}^{N} \int_{\Omega_{j}} \eta_{j} d x d y=0 \text { and } \\
|\Omega|^{-1} \sum_{j=0}^{N} \int_{\Omega_{j}}\left(\Phi_{j}+i \omega_{n} m_{j} \eta_{j}\right) d x d y=0 .
\end{gathered}
$$

Proof. The above properties can be easily shown by appropriately testing the variational form. Due to the homogeneous Neumann conditions for $\Phi_{0}$ on $\Gamma$, the fact that the plate is freely floating and the form of the interface conditions, every constant function is admissible as a weight function in the variational form. Selecting $\chi_{j}=w_{j}=w_{0}=1$, in (3.12) results in

$$
i \omega \sum_{j=1}^{N} \int_{\Omega_{j}} \eta_{j} d x d y+\omega^{2} \int_{\Omega_{0}} \Phi_{0} d x d y+\sum_{j=1}^{N} \int_{\Gamma_{j}}\left(B_{j} \nabla \Phi_{j} \cdot \mathbf{n}_{j}+B_{0} \nabla \Phi_{0} \cdot \mathbf{n}_{0}\right) d s=0
$$


The free surface elevation in domain $\Omega_{0}$ is $\eta_{0}=-i \omega \Phi_{0}$. Thus, using interface conditions (2.9) and the fact that $\mathbf{n}_{j}=-\mathbf{n}_{0}$, it is

$$
i \omega \sum_{j=1}^{N} \int_{\Omega_{j}} \eta_{j} d x d y-\frac{\omega}{i} \int_{\Omega_{0}} \eta_{0} d x d y=0
$$

which implies (4.1). For the derivation of (4.2), note that when $\chi_{j}=w_{j}=w_{0}=1$ Eq. (3.12) becomes

$$
\sum_{j=1}^{N}\left[(1-i \omega) \int_{\Omega_{j}} \eta_{j} d x d y-\omega^{2} \int_{\Omega_{j}} m_{j} \eta_{j} d x d y+i \omega \int_{\Omega_{j}} \Phi_{j} d x d y\right]-i \omega \int_{\Omega_{0}} \eta_{0} d x d y=0
$$

Adding the term $(1-i \omega) \int_{\Omega_{0}} \eta_{0} d x d y-\left(i \omega+\omega^{2}\right) \int_{\Omega_{0}} \Phi_{0} d x d y=0$, and rearranging the integrals in the series, results to

$$
\sum_{j=0}^{N} \int_{\Omega_{j}} \Phi_{j} d x d y=-i \omega_{n} \sum_{j=1}^{N} \int_{\Omega_{j}} m_{j} \eta_{j} d x d y
$$

Eq. (4.2) now follows since $m_{0}=0$.

It is worth mentioning that when floating plates are present in the basin, the upper surface elevation is not continuous at the interface between the plates and free water regions. These discontinuities occur since different differential operators govern the evolution of the field in the hydroelastic and free water regions. According now to (4.1), the mean value of the upper surface elevation (including these finite jumps) vanishes.

\subsection{Eigenfrequency Spectrum and Orthogonality Conditions}

This subsection is devoted to the proof of the following proposition regarding the distribution of eigenvalues for the problem considered and the derivation of orthogonality conditions for the eigenfunctions. In order to derive the orthogonality conditions, the variational form in eigenstate $p$ is tested with the admissible weight functions $\left\{\eta_{j}^{(q)}, i \Phi_{j}^{(q)}, i \Phi_{0}^{(q)}\right\}, j=1,2, \ldots, N$, while in eigenstate $q$ the weight functions $\left\{\eta_{j}^{(p)}, i \Phi_{j}^{(p)}, i \Phi_{0}^{(p)}\right\}$ are used. At all eigenstates functions $\eta_{j}, \Phi_{j}, \Phi_{0}$ are real since the standing wave solutions pursued and Eqs. (3.2-3.4) imply that the surface elevation and velocity potential are real and imaginary (or vice versa) respectively, without any loss of generality. The process of the proof for these properties will furthermore reveal several other important inequalities that the hydroelastic fields satisfy.

\section{PROPOSITION 2}

(i) The eigenvalues are real and symmetrically placed with respect to the imaginary axis 
(ii) The eigensolution corresponding to $\omega_{0}=0$ is $\eta_{j} \equiv 0$ in $\Omega_{j}$ for $j=1,2, \ldots, N$ and $\Phi_{j} \equiv c_{j}$ in $\Omega_{j}$ for $j=0,1,2, \ldots, N$, where $c_{j}$ are constants

(iii) (Orthogonality conditions) Given any two eigenstates $p \in \mathbb{N}$ and $q \in \mathbb{N}$ of the hydroelastic system, where $p \neq q$, it is

$$
\begin{gathered}
\left(\omega_{p}+\omega_{q}\right)\left(\int_{\Omega_{0}} \Phi_{0}^{(q)} \Phi_{0}^{(p)} d x d y+\sum_{j=1}^{N} \int_{\Omega_{j}} m_{j} \eta_{j}^{(q)} \eta_{j}^{(p)} d x d y\right) \\
+\sum_{j=1}^{N} \int_{\Omega_{j}}\left(\eta_{j}^{(p)} \Phi_{j}^{(q)}+\Phi_{j}^{(p)} \eta_{j}^{(q)}\right) d x d y=0 \\
\omega_{p} \omega_{q}\left(\int_{\Omega_{0}} \Phi_{0}^{(q)} \Phi_{0}^{(p)} d x d y+\sum_{j=1}^{N} \int_{\Omega_{j}} m_{j} \eta_{j}^{(q)} \eta_{j}^{(p)} d x d y\right)-\int_{\Omega_{0}} B_{0} \nabla \Phi_{0}^{(q)} \cdot \nabla \Phi_{0}^{(p)} d x d y \\
-\sum_{j=1}^{N}\left[a_{j}\left(\eta_{j}^{(q)}, \eta_{j}^{(p)}\right)+\int_{\Omega_{j}} \eta_{j}^{(q)} \eta_{j}^{(p)} d x d y+\int_{\Omega_{j}} B_{j} \nabla \Phi_{j}^{(q)} \cdot \nabla \Phi_{j}^{(p)} d x d y\right]=0
\end{gathered}
$$

Proof. Regarding the existence of real eigenvalues, testing the variational form (3.12) with the admissible functions $\bar{\chi}_{j}=\bar{\eta}_{j}, \bar{w}_{j}=\bar{\Phi}_{j}$ and $\bar{w}_{0}=\bar{\Phi}_{0}$, results to

$$
\begin{aligned}
\omega^{2}\left[\left\|\Phi_{0}\right\|_{L^{2}\left(\Omega_{0}\right)}^{2}+\sum_{j=1}^{N}\left\|\sqrt{m_{j}} \eta_{j}\right\|_{L^{2}\left(\Omega_{j}\right)}^{2}\right]-i \omega \sum_{j=1}^{N}\left[\int_{\Omega_{j}}\left(\bar{\eta}_{j} \Phi_{j}-\bar{\Phi}_{j} \eta_{j}\right) d x d y\right] \\
-\left\|\sqrt{B_{0}} \nabla \Phi_{0}\right\|_{L^{2}\left(\Omega_{0}\right)}^{2}-\sum_{j=1}^{N}\left[a_{j}\left(\bar{\eta}_{j}, \eta_{j}\right)+\left\|\eta_{j}\right\|_{L^{2}\left(\Omega_{j}\right)}^{2}+\left\|\sqrt{B_{j}} \nabla \Phi_{j}\right\|_{L^{2}\left(\Omega_{j}\right)}^{2}\right]=0
\end{aligned}
$$

where $\|\cdot\|_{L^{2}\left(\Omega_{j}\right)}$ is the $L^{2}$ norm of a function defined in $\Omega_{j}$. Since it is

$$
i \sum_{j=1}^{N}\left[\int_{\Omega_{j}}\left(\bar{\eta}_{j} \Phi_{j}-\bar{\Phi}_{j} \eta_{j}\right) d x d y\right]=2 \sum_{j=1}^{N}\left[\int_{\Omega_{j}}\left(\operatorname{Re} \Phi_{j} \operatorname{Im} \eta_{j}-\operatorname{Re} \eta_{j} \operatorname{Im} \Phi_{j}\right) d x d y\right] \in \mathbb{R}
$$

Eq. (4.7) is a quadratic polynomial with real coefficients, of the form $\alpha \omega^{2}+\beta \omega-\gamma=0$. For $\omega$ to be real it suffices to show that the discriminant is positive or zero, that is $\Delta=\beta^{2}+4 \alpha \gamma \geq 0$. This condition, since $\alpha=\left\|\Phi_{0}\right\|_{L^{2}\left(\Omega_{0}\right)}^{2}+\sum_{j=1}^{N}\left\|\sqrt{m_{j}} \eta_{j}\right\|_{L^{2}\left(\Omega_{j}\right)}^{2} \geq 0$, reduces to $\gamma \geq 0$. This last inequality can be easily verified by using the strong ellipticity of $a_{j}$ for a plate bending problem on a Winkler type foundation, as it is $K_{j}>0$ and $0<v_{j}<1 / 2$, thus [24]

$$
\begin{aligned}
& \sum_{j=1}^{N}\left(a_{j}\left(\bar{\eta}_{j}, \eta_{j}\right)+\int_{\Omega_{j}} \bar{\eta}_{j} \eta_{j} d x d y\right) \geq \\
& \sum_{j=1}^{N}\left\|\eta_{j}\right\|_{L^{2}\left(\Omega_{j}\right)}^{2}+\sum_{j=1}^{N}\left[\left\|\sqrt{K_{j} v_{j}} \Delta \eta_{j}\right\|_{L^{2}\left(\Omega_{j}\right)}^{2}+\operatorname{essinf}_{\Omega_{j}}\left\{K_{j}\left(1-v_{j}\right)\right\}\left|\eta_{j}\right|_{H^{2}\left(\Omega_{j}\right)}^{2}\right] \geq 0
\end{aligned},
$$


where $\left|\eta_{j}\right|_{H^{2}\left(\Omega_{j}\right)}$ is the $H^{2}\left(\Omega_{j}\right)$ semi-norm. Now, the result follows directly from the $L^{2}$ products appearing in (4.8) and the definition of the respective norms.

At all eigenstates, when $\omega \neq 0$, using Eqs (3.3), (3.4) and the Green-Gauss theorem it is

$$
\begin{aligned}
& i \omega \sum_{j=1}^{N}\left[\int_{\Omega_{j}}\left(\bar{\eta}_{j} \Phi_{j}-\bar{\Phi}_{j} \eta_{j}\right) d x d y\right]=\sum_{j=1}^{N}\left[\int_{\Omega_{j}}\left(\Phi_{j} \nabla \cdot\left(B_{j} \nabla \bar{\Phi}_{j}\right)+\bar{\Phi}_{j} \nabla \cdot\left(B_{j} \nabla \Phi_{j}\right)\right) d x d y\right]= \\
& -2 \sum_{j=1}^{N}\left\|\sqrt{B_{j}} \nabla \Phi_{j}\right\|_{L^{2}\left(\Omega_{j}\right)}^{2}+\sum_{j=1}^{N} \int_{\Gamma_{j}}\left(\bar{\Phi}_{j} B_{j} \nabla \Phi_{j} \cdot \mathbf{n}_{j}+\Phi_{j} B_{j} \nabla \bar{\Phi}_{j} \cdot \mathbf{n}_{j}\right) d s= \\
& -2 \sum_{j=1}^{N}\left\|\sqrt{B_{j}} \nabla \Phi_{j}\right\|_{L^{2}\left(\Omega_{j}\right)}^{2}-\sum_{j=1}^{N} \int_{\Gamma_{j}}\left(\bar{\Phi}_{0} B_{0} \nabla \Phi_{0} \cdot \mathbf{n}_{0}+\Phi_{0} B_{0} \nabla \bar{\Phi}_{0} \cdot \mathbf{n}_{0}\right) d s= \\
& -2 \sum_{j=1}^{N}\left\|\sqrt{B_{j}} \nabla \Phi_{j}\right\|_{L^{2}\left(\Omega_{j}\right)}^{2}-2\left\|\sqrt{B_{0}} \nabla \Phi_{0}\right\|_{L^{2}\left(\Omega_{0}\right)}^{2}+2 \omega^{2}\left\|\Phi_{0}\right\|_{L^{2}\left(\Omega_{0}\right)}^{2}
\end{aligned}
$$

Equation (4.6) then becomes

$$
\begin{aligned}
& \omega^{2}\left[\sum_{j=1}^{N}\left\|\sqrt{m_{j}} \eta_{j}\right\|_{L^{2}\left(\Omega_{j}\right)}^{2}-\left\|\Phi_{0}\right\|_{L^{2}\left(\Omega_{0}\right)}^{2}\right]+ \\
& \left\|\sqrt{B_{0}} \nabla \Phi_{0}\right\|_{L^{2}\left(\Omega_{0}\right)}^{2}-\sum_{j=1}^{N}\left[a_{j}\left(\bar{\eta}_{j}, \eta_{j}\right)+\left\|\eta_{j}\right\|_{L^{2}\left(\Omega_{j}\right)}^{2}-\left\|\sqrt{B_{j}} \nabla \Phi_{j}\right\|_{L^{2}\left(\Omega_{j}\right)}^{2}\right]=0
\end{aligned}
$$

Since it has already been established that all eigenvalues are real, the above equation suggests that eigenvalues are symmetrically placed with respect to the imaginary axis.

Furthermore, since $\left\|\Phi_{0}\right\|_{L^{2}\left(\Omega_{0}\right)}^{2}=\omega^{-2}\left\|\eta_{0}\right\|_{L^{2}\left(\Omega_{0}\right)}^{2}$, equation (4.9) can be written as

$$
\omega^{2}\left[\sum_{j=1}^{N}\left\|\sqrt{m_{j}} \eta_{j}\right\|_{L^{2}\left(\Omega_{j}\right)}^{2}\right]=\sum_{j=1}^{N} a_{j}\left(\bar{\eta}_{j}, \eta_{j}\right)+\sum_{j=0}^{N}\left\|\eta_{j}\right\|_{L^{2}\left(\Omega_{j}\right)}^{2}-\sum_{j=0}^{N}\left\|\sqrt{B_{j}} \nabla \Phi_{j}\right\|_{L^{2}\left(\Omega_{j}\right)}^{2},
$$

which implies that at all eigenstates, when $\omega \neq 0$, it is

$$
\sum_{j=1}^{N} a_{j}\left(\bar{\eta}_{j}, \eta_{j}\right)+\sum_{j=0}^{N}\left\|\eta_{j}\right\|_{L^{2}\left(\Omega_{j}\right)}^{2}>\sum_{j=0}^{N}\left\|\sqrt{B_{j}} \nabla \Phi_{j}\right\|_{L^{2}\left(\Omega_{j}\right)}^{2}
$$

When $\omega=0$, Eq. (4.6) becomes

$$
\left\|\sqrt{B_{0}} \nabla \Phi_{0}\right\|_{L^{2}\left(\Omega_{0}\right)}^{2}+\sum_{j=1}^{N}\left[a_{j}\left(\bar{\eta}_{j}, \eta_{j}\right)+\left\|\eta_{j}\right\|_{L^{2}\left(\Omega_{j}\right)}^{2}+\left\|\sqrt{B_{j}} \nabla \Phi_{j}\right\|_{L^{2}\left(\Omega_{j}\right)}^{2}\right]=0
$$

Inequality (4.8) now implies $\eta_{j}=0$. The $L^{2}$ norms of the velocity potential gradient being zero is equivalent to $\Phi_{j}=c_{j}$, where $c_{j} j=0,1,2, \ldots, N$ are constants. Since the 
velocity potentials are defined up to a constant, the zero solution when $\omega=0$ is achieved if the quotient spaces $\Phi_{j} \in H_{\mathbb{C}}^{1}(\Omega)$ are considered.

Testing the variational form in eigenstate $p$ with the admissible weight functions $\left\{\eta_{j}^{(q)}, i \Phi_{j}^{(q)}, i \Phi_{0}^{(q)}\right\}, j=1,2, \ldots, N$, and the variational form in eigenstate $q$ with the weight functions $\left\{\eta_{j}^{(p)}, i \Phi_{j}^{(p)}, i \Phi_{0}^{(p)}\right\}$ results, due to the symmetry of the bilinear functionals $a_{j}$, into two quadratic expressions of the form

$$
A \omega_{p}^{2}+B \omega_{p}+J=0, A \omega_{q}^{2}+B \omega_{q}+J=0
$$

are derived, having the same coefficients

$$
\begin{gathered}
A=\int_{\Omega_{0}} \Phi_{0}^{(q)} \Phi_{0}^{(p)} d x d y+\sum_{j=1}^{N} \int_{\Omega_{j}} m_{j} \eta_{j}^{(q)} \eta_{j}^{(p)} d x d y, \\
B=\sum_{j=1}^{N} \int_{\Omega_{j}}\left(\eta_{j}^{(p)} \Phi_{j}^{(q)}+\Phi_{j}^{(p)} \eta_{j}^{(q)}\right) d x d y \text { and } \\
J=\sum_{j=1}^{N}\left[a_{j}\left(\eta_{j}^{(q)}, \eta_{j}^{(p)}\right)+\int_{\Omega_{j}} \eta_{j}^{(q)} \eta_{j}^{(p)} d x d y+\int_{\Omega_{j}} B_{j} \nabla \Phi_{j}^{(q)} \cdot \nabla \Phi_{j}^{(p)} d x d y\right] . \\
+\int_{\Omega_{0}} B_{0} \nabla \Phi_{0}^{(q)} \cdot \nabla \Phi_{0}^{(p)} d x d y
\end{gathered}
$$

and two positive roots $\omega_{p}, \omega_{q}$. Vieta's formulae for the sum and product of the quadratic polynomial roots with coefficients $A, B, J$, yield the desired result.

\subsection{Energy Conservation and the Rayleigh Quotient}

A second important property of the hydroelastic interaction model presented above is the absence of energy dissipation mechanisms. This property is linked to the presence of a conserved quantity (energy) that can be used for the definition of a Rayleigh-type quotient characterizing the eigenvalues of the system. Such a result can be directly derived from Eq. (4.10) as

PROPOSITION 3 (Rayleigh Quotient) At all eigenstates $p \in \mathbb{N}$ it is

$$
\omega_{p}^{2}=\frac{\sum_{j=1}^{N} a_{j}\left(\bar{\eta}_{j}, \eta_{j}\right)+\sum_{j=0}^{N}\left[\left\|\eta_{j}\right\|_{L^{2}\left(\Omega_{j}\right)}^{2}-\left\|\sqrt{B_{j}} \nabla \Phi_{j}\right\|_{L^{2}\left(\Omega_{j}\right)}^{2}\right]}{\sum_{j=1}^{N}\left\|\sqrt{m_{j}} \eta_{j}\right\|_{L^{2}\left(\Omega_{j}\right)}^{2}} .
$$

In concluding this section it is interesting to note the similarities of the present eigenvalue problem with Sturm-Liouville systems. Extensive numerical evidence shows that the eigenstates behave as stationary points of the Rayleigh quotient, similarly as in the case of Sturm-Liouville systems. 


\section{Finite Element Solution of a simplified Eigenproblem}

Several properties of the hydroelastic eigenstates, as predicted by the proposed model, have been studied qualitatively in the previous section. A parametric analysis with respect to geometric and plate material characteristics of the floating plate/basin system is expected to provide further insight. The analytical solution of the hydroelastic problem under consideration is very difficult or even impossible for involved geometries or variable bathymetry basins. Therefore, a finite element procedure for determining the hydroelastic eigenfrequencies and eigenmodes will be adopted.

Let $\quad \eta_{j}^{h} \in V^{h}, \quad \Phi_{j}^{h} \in W_{j}^{h} \subset H^{1}\left(\Omega_{j} ; \mathbb{C}\right) \quad$ and $\quad \Phi_{0}^{h} \in W_{0}^{h} \subset H^{1}\left(\Omega_{0} ; \mathbb{C}\right)$. The case $V^{h} \subset H^{2}\left(\Omega_{0} ; \mathbb{C}\right)$, that could lead to conforming approximations, is demanding for 2D problems due to the presence of the fourth order operator. In 1D cases, conforming Hermite interpolation is straightforward to apply for the specific hydroelastic model [26]. The restriction of the approximation functions in a specific hydroelastic finite element $\mathrm{K}$ (in hydroelastic region $j$ ) can be written as $\left.\eta_{j}^{h}\right|_{\mathrm{K}}=\sum_{a=1}^{S_{P}} H_{j}^{(a)} \zeta_{j}^{(a)}$ and $\left.\Phi_{j}^{h}\right|_{\mathrm{K}}=\sum_{a=1}^{S_{W}} L_{j}^{(a)} \Phi_{j}^{(a)}$, where $H_{j}^{(a)}$ are shape functions for the plate deformation and $L_{j}^{(a)}$ are typical Lagrange shape functions for the velocity potential. The natural numbers $S_{P}$ and $S_{W}$ indicate the number of shape functions adopted for the two fields and thus characterise the order of approximation of the hydroelastic element. The nodal unknown quantities for the plate $\zeta_{j}^{(a)}$ include deflections and their derivatives. In the free water region, where the governing model is the D'Alembert wave equation, the FE approximation is $\left.\Phi_{0}^{h}\right|_{\mathrm{K}}=\sum_{a=1}^{S_{W}} L_{0}^{(a)} \Phi_{0}^{(a)}$ based on Lagrangian shape functions. A quasi-uniform triangulation of $\Omega$, where elements in contact with the interfaces $\Gamma_{j}$ have nodes on the interface, is assumed. The above presented approximations, using variational form (3.12) and the standard Galerkin method, produce the local hydroelastic finite element matrices

$$
\begin{gathered}
\mathbf{m}_{\mathrm{K}(j)}=\mathbf{m}_{\alpha \beta}=\left(\omega^{h}\right)^{2} \int_{\Omega_{j}} m_{j} H_{j}^{(\alpha)} H_{j}^{(\beta)} d x d y, \\
\mathbf{c}_{\mathrm{K}(j)}=\mathbf{c}_{\alpha \beta}=-i \omega^{h} \int_{\Omega_{j}}\left(H_{j}^{(\alpha)} L_{j}^{(\beta)}-L_{j}^{(\alpha)} H_{j}^{(\beta)}\right) d x d y, \\
\mathbf{k}_{\mathrm{K}(j)}=\mathbf{k}_{\alpha \beta}=-a_{j}\left(H_{j}^{(\alpha)}, H_{j}^{(\beta)}\right)-\int_{\Omega_{j}} H_{j}^{(\alpha)} H_{j}^{(\beta)} d x d y-\int_{\Omega_{j}} B_{j} \nabla L_{j}^{(\alpha)} \cdot \nabla L_{j}^{(\beta)} d x d y,
\end{gathered}
$$

and the following standard local matrices in the free water region

$$
\mathbf{m}_{\mathrm{K}(0)}=\mathbf{m}_{\alpha \beta}=\left(\omega^{h}\right)^{2} \int_{\Omega_{0}} L_{0}^{(\alpha)} L_{0}^{(\beta)} d x d y, \mathbf{k}_{\mathrm{K}(0)}=\mathbf{k}_{\alpha \beta}=-\int_{\Omega_{0}} B_{0} \nabla L_{0}^{(\alpha)} \cdot \nabla L_{0}^{(\beta)} d x d y,
$$


where $\omega^{h}$ is the eigenfrequency approximation. Combining the local contributions and formulating a global system of FE equations leads to a Quadratic Eigenvalue Problem (QEP) $[25,27]$ of the form

$$
\left(\left(\omega^{h}\right)^{2} \mathbf{M}+\omega^{h} \mathbf{C}+\mathbf{K}\right) \mathbf{u}^{h}=\mathbf{0},
$$

where, $\mathbf{M}$ is real and has contributions from $\mathbf{m}_{\mathrm{K}(j)}, \mathbf{m}_{\mathrm{K}(0)}$ over all elements $\mathrm{K}$ in $\Omega$, C has contributions from $\mathbf{c}_{\mathrm{K}(j)}$ in all $\Omega_{j}$ and $\mathbf{K}$ is real with contributions from $\mathbf{k}_{\mathrm{K}(j)}, \mathbf{k}_{\mathrm{K}(0)}$ in $\Omega$. Since $\Phi_{j}$ are not represented in $\mathbf{M}$, the matrix is positive semidefinite. Global matrix $\mathbf{C}$ is singular as well (since it is only defined in the hydroelastic regions) and furthermore it is $\mathbf{C}^{T}=c_{\beta \alpha}=-c_{\alpha \beta}=-\mathbf{C}$. The QEP has thus a singular mass-type matrix and skew-symmetric damping-type matrix. For this type of problems, the eigenvalues are real and there exist both finite and infinite in measure eigenvalues $[25,27]$. Both these facts are reflected in the analysis of the continuous system presented in section 4.2. The properties of the discrete system are a direct consequence of the skew symmetric form in (4.7), produced by the terms with first time derivatives in the time domain problem. Such terms do not correspond to energy loses and therefore conservation of energy, compatible with the fact that no dissipative mechanisms are present in the model, occurs. This property is then reflected in the real valued spectrum of the system. Since $\mathbf{M}$ is singular, the analysis can be performed in terms of the reverse polynomial [25], setting $\lambda=\omega^{-1}$

$$
Q(\lambda)=\lambda^{2} \mathbf{K}+\lambda \mathbf{C}+\mathbf{M}=\mathbf{O} .
$$

Using the reverse polynomial, the infinite measure eigenfrequencies are mapped to the zero values of $\lambda$. The reverse polynomial can be solved directly in MATLAB $\AA$ using the polynomial eigenvalue solver 'polyeig', or by linearization. In the following, the above FE based numerical strategy will be adopted, utilizing the HELFEM hydroelastic elements introduced in [26] for plates of infinite width. Finite Elements for simulations in two horizontal dimensions, based on nonconforming approximations will be the subject of a forthcoming study.

\section{FE Convergence and Parametric Analysis}

The effect of several parameters, present in the hydroelastic eigenproblem, will be studied, using the hydroelastic elements HELFEM, introduced in [26]. In order to simplify the analysis and isolate the effect that specific factors have on the hydroelastic response, basins of constant depth and a single floating plate of infinite width will be initially considered. The convergence characteristics of the HELFEM elements will be studied first. A basin of total length $L=1000 \mathrm{~m}$ is considered. The floating plate has stiffness $E=5 \mathrm{GPa}$, Poisson's ratio $v=0.3$ and density 
$\rho=900 \mathrm{~kg} / \mathrm{m}^{3}$. These values are close to the properties of sea ice [12], however the analysis is also relevant to VLFS, the response of which is governed by the same model [28]. In all cases hereafter the plate's thickness is $\tau=1 \mathrm{~m}$, gravity acceleration is $g=9.81 \mathrm{~m} / \mathrm{sec}^{2}$ and the density of water is $\rho_{w}=1000 \mathrm{~kg} / \mathrm{m}^{3}$. A floating plate of span $l_{P}=200 \mathrm{~m}$ is located at the middle of the pond. The first and sixth eigenfrequency is calculated using the hydroelastic finite elements $\operatorname{HELFEM}(3,2)$ and $\operatorname{HELFEM}(5,4)[26]$. Element $\operatorname{HELFEM}(3,2)$ has standard cubic Hermite interpolation of $3^{\text {rd }}$ degree for the deflection and quadratic Lagrange interpolation for the velocity potential. Element $\operatorname{HELFEM}(5,4)$ features Hermite interpolation of $5^{\text {th }}$ degree for the deflections and quartic Lagrange polynomials for the velocity potential. For this specific problem an analytical solution was also pursued using a sixth order ordinary differential equation for the velocity potential in the hydroelastic region [22] and subsequently applying interface and freely floating plate conditions. In particular, following the analysis adopted by J.J. Stoker [22] and also by I.V. Sturova [12] and Meylan et al. [19], the velocity potential in a hydroelastic region $j$ can be expressed in the form

$$
\Phi_{j}(x)=\sum_{n=1}^{6} A_{n} e^{\lambda_{n} x}
$$

where $\lambda_{n}$ are the roots of the polynomial $K \lambda^{6}+\left(1-\omega^{2} M\right) \lambda^{2}+(B-M)^{-1} \omega^{2}=0$. The velocity potential outside the hydroelastic region is of the form

$$
\Phi(x)=D_{1} \cos (\omega x / \sqrt{B})+D_{2} \sin (\omega x / \sqrt{B}) .
$$

Using the interface conditions (3.5b), the zero bending moment and shear force conditions at the end points of the floating plate and the zero velocity conditions at the end points of the basin, a homogeneous system for the unknowns $A_{n}, D_{1}, D_{2}$ is derived. For nontrivial solutions, this approach involves the calculation of a determinant that leads to a transcendental equation for the eigenfrequencies and the eigenfunctions. This approach is very efficient for one floating plate but becomes more involved if two or more plates are considered. Furthermore it can only be applied to constant depth basins. The analytical solutions derived are used to establish convergence rates for the finite element scheme adopted and for validation. For the example analysed in figure 3 regarding the eigenfrequencies, a solution with 50 elements is the same as the exact one within machine precision. The observed convergence rates, based on comparison against the analytical solution, calculated by using 5, 10, 15, 20 and 25 elements in total, are plotted using double logarithmic scale in Fig. 3. The observed convergence rates are very close to the expected, theoretical ones, (4 for $\operatorname{HELFEM}(3,2)$ and $8 \operatorname{HELFEM}(5,4)$ ), based on the selected order of interpolation [24]. Furthermore, as expected, the approximation is found to deteriorate as the mode number increases, although the errors obtained are very small even with coarse meshes. HELFEM $(5,4)$ for the first eigenfrequency provides results of machine 
precision even for very coarse meshes. The expected rates have been also obtained for the eigenmodes.
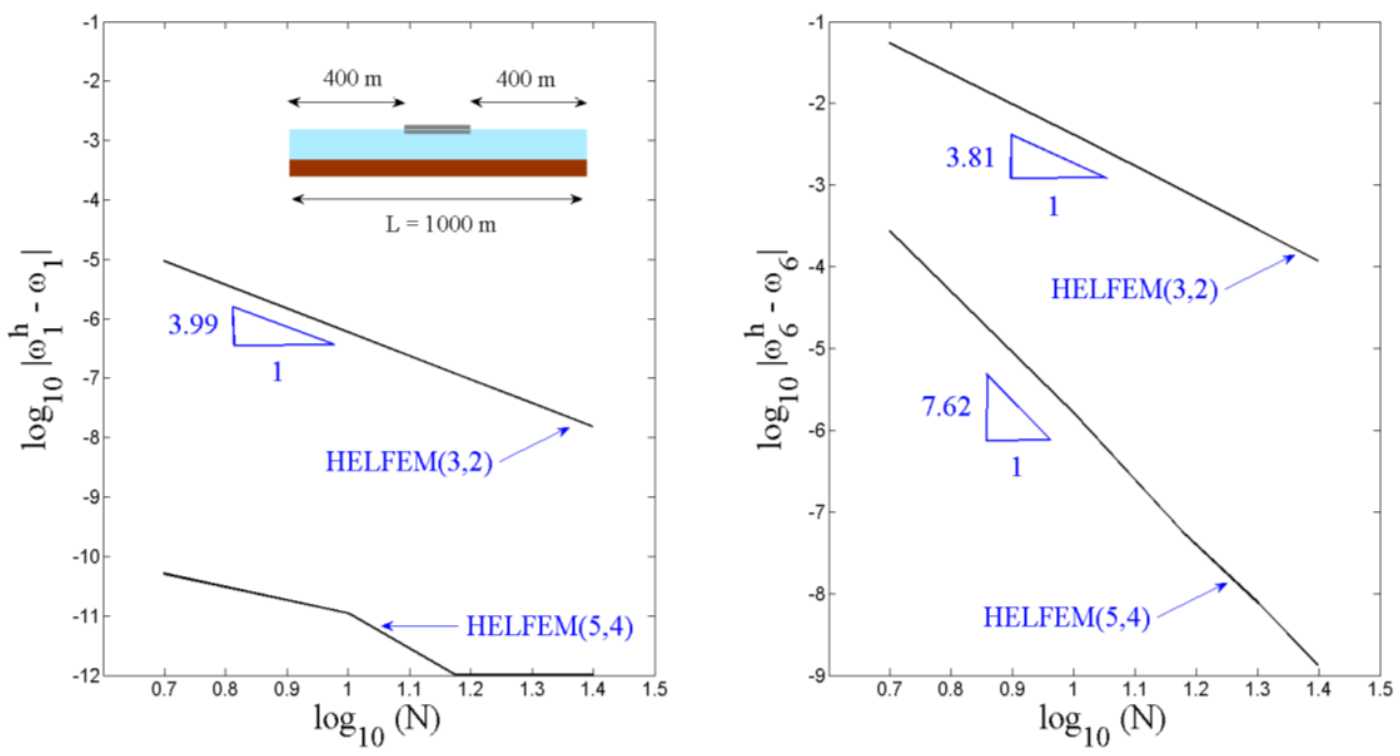

Figure 3. Convergence rates for Finite Elements $\operatorname{HELFEM}(3,2)$ and $\operatorname{HELFEM}(5,4)$.

In the simplified setting introduced, using the hydroelastic elements $\operatorname{HELFEM}(3,2)$, the effect of the plate's span, position, stiffness, mass and draft will be first analysed. Furthermore, examples regarding multiple floating bodies and shallow basins of variable bathymetry will be presented. The influence of all these parameters will be quantified using two indicators. The first indicator is the $L^{2}$ norm of the difference between the upper surface elevation in the presence and absence of a floating body, over the respective norm of the basin without the body. For the basin without the floating plate this function at eigenstate $n$ is denoted as $H_{n}$, while for basin with the floating plate, the upper surface elevation, including the plate deflection at the region of hydroelastic interaction, is denoted as $\eta_{n}$. The first indicator is thus

$$
\mathrm{Y}_{n}=\frac{\left\|\eta_{n}-H_{n}\right\|_{L^{2}(\Omega)}}{\left\|H_{n}\right\|_{L^{2}(\Omega)}},
$$

and describes the influence of the floating plate on the eigenfunctions. The second indicator is the ratio of the hydroelastic system eigenfrequency $\omega_{n}$ over the respective eigenfrequency of the pond without the floating plate, denoted here as $\Omega_{n}$. The eigenfrequencies and eigenfunctions for a basin of length $L$ and constant depth $B$ are

$$
\Omega_{n}=n \pi \frac{\sqrt{B}}{L}, H_{n}(x)=C_{n} \cos \left(\frac{n \pi x}{L}\right),
$$

where $C_{n}$ is a scaling constant. 


\subsection{Effect of Plate Span and Position}

First the effect of the plate's span in a basin of total length $L=1000 \mathrm{~m}$ is considered. The floating plate has the same as above material properties. The first six eigenstates for a basin of depth $2 \mathrm{~m}$ with a floating plate of length $l_{P}=400 \mathrm{~m}$, are shown in Fig 4. The plate's midpoint coincides with the midpoint of the basin. In all cases presented henceforth the horizontal coordinate is translated such that point zero coincides with the left edge of the plate. The upper surface elevation of the basin without the floating plate is depicted as a blue dashed line. All eigenmodes have been scaled so that they have maximum amplitude equal to one in the dimensional setting. It is evident that the presence of the floating body produces differences in the eigenfunction profile and that these differences become more significant at higher eigenmodes. Note that the basin considered in this example is extremely shallow ( $2 \mathrm{~m}$ depth). However, this configuration can be related to applications of floating Photovoltaic (PV) platforms in shallow ponds [13]. It is interesting to note that the upper surface elevation at the edges of the plate has not the same value as the upper surface elevation at the water next to the edges. In the cases considered this jump in the upper surface elevation/plate deflection has very small values and is not visible without zooming at the specific locations. Finally, the small depth of the basin ensures that the long wave approximation adopted is valid for even higher modes.
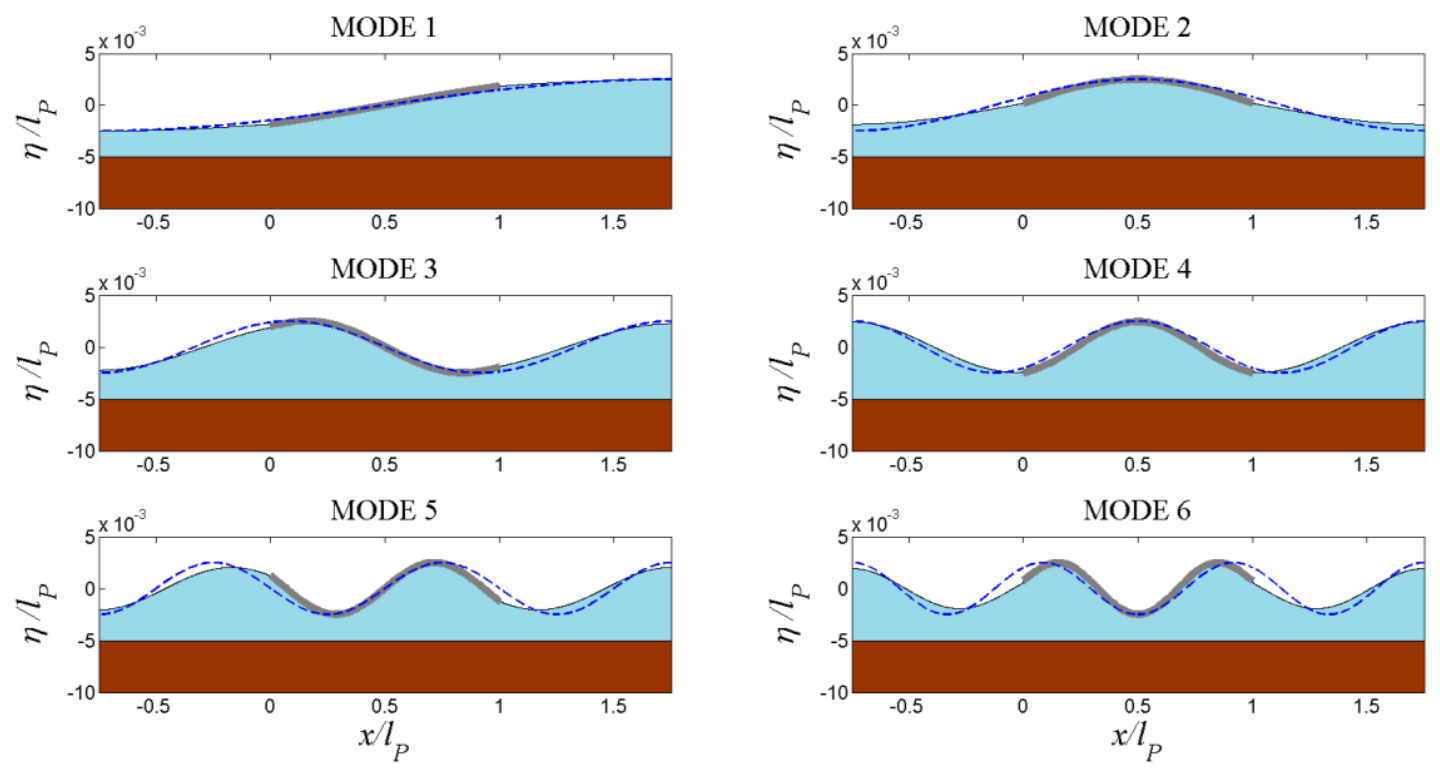

Figure 4. Eigenstates for a floating plate of span $400 \mathrm{~m}$ at the middle of a closed shallow basin (1000m length and $2 \mathrm{~m}$ deep). The mode of the basin without the plate is plotted as blue dashed line.

The effect of the plate span is studied in Figs. 5 and 6. In all cases the plate's midpoint is located at the middle of the basin. The case of the very shallow basin $(2 \mathrm{~m})$ is considered along with a basin of $3 \mathrm{~m}$ and one of $4 \mathrm{~m}$ depth. The length of the floating plate $l_{P}$ is increased up to the point that the whole pond is covered. Figure 5 shows the 
first indicator as a function of the nondimensional plate span $l_{P} / L$. It is thus representative of the plate's span effect on the eigenfunctions. It is evident that as the depth increases the effect of the floating plate in the pond becomes less significant. Higher modes are affected more by the presence of the plate. At the same time, the effect of the plate is more intense when the plate covers only a portion of the basin.
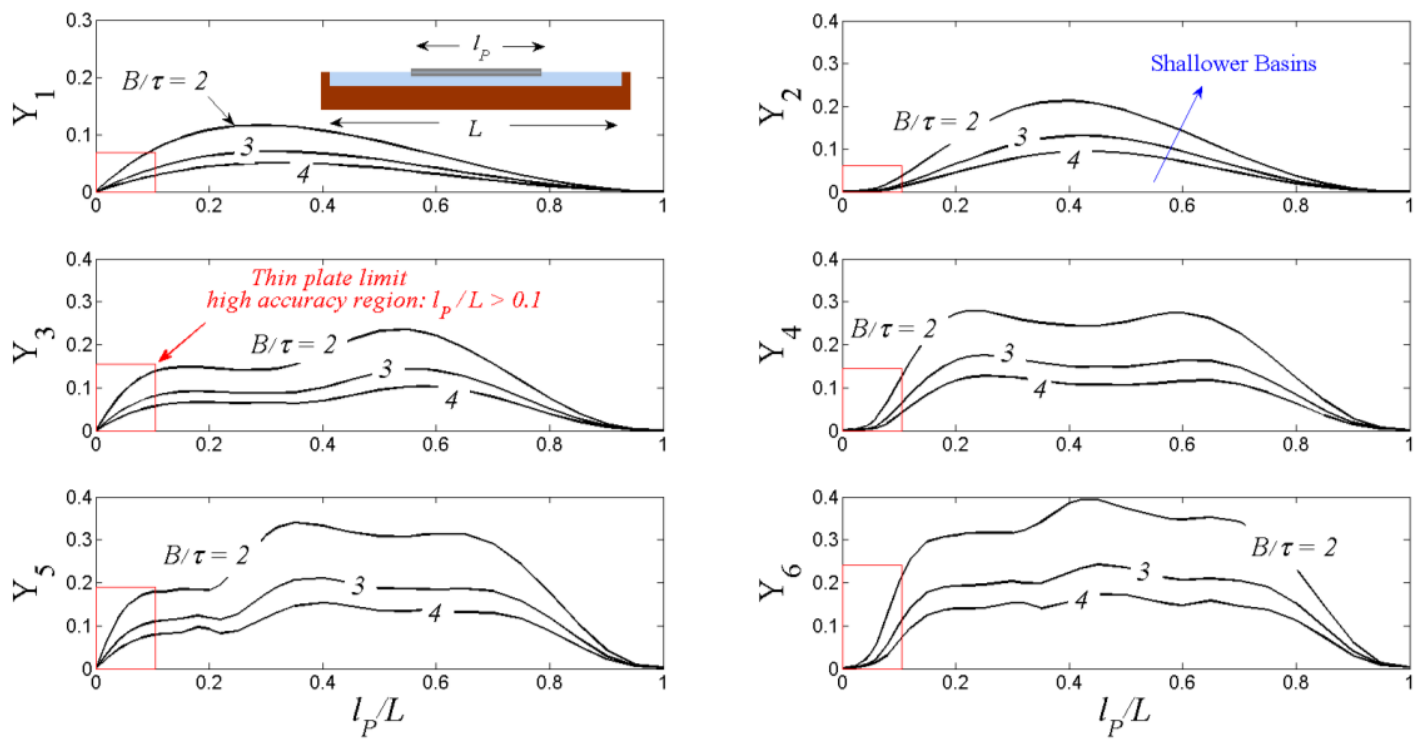

Figure 5. Effect of the floating plate span on the upper surface elevation - plate deflection in a shallow basin of depth $B \mathrm{~m}$. The plate thickness is $\tau=1 \mathrm{~m}$.
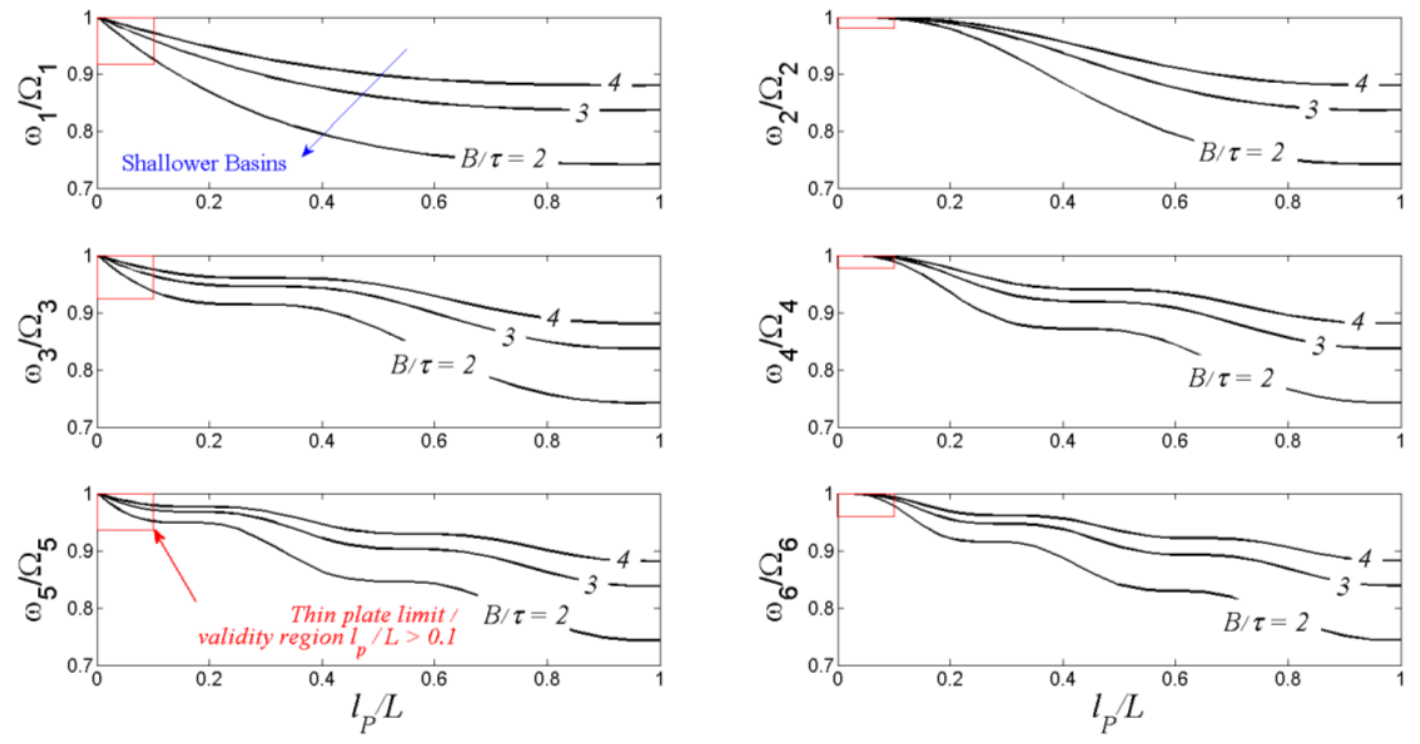

Figure 6. Effect of the floating plate span on the eigenfrequencies of a shallow basin having depth $B \mathrm{~m}$. The plate thickness is $\tau=1 \mathrm{~m}$.

As a general rule it can be stated that the effect is more significant when the plate span is approximately half the basin length, although the actual span that maximises the first indicator depends on the specific mode considered. In all cases, if the plate 
increases to the point that it covers the whole basin, the differences in the upper surface elevation between the basin without the plate and the basin with the floating plate become almost zero. Finally, for values of the ratio $l_{P} / L<0.1$, since the thickness of the plate is $\tau=1 \mathrm{~m}$, the floating plate cannot be classified as thin $\left(\tau / l_{P}<0.01\right)$ and the results gradually become inaccurate as $l_{P} / L$ decreases. The problematic region is depicted using a red frame in Figs. 5 and 6. Figure 6, shows the effect of the plate span on the eigenfrequencies of the system. The effect here has the reverse trend when compared to figure 5. The eigenfrequenies are affected more as the span of the floating plate increases relative to the length of the basin. Again, shallower basins are affected more. A maximum reduction of approximately $25 \%$ is observed for the shallower basin, while the maximum reduction is approximately $17 \%$ when $B / \tau=3$ and $12 \%$ when $B / \tau=4$.

REMARK: When a floating plate of relatively small stiffness spans the whole basin it has been observed that the eigenfunctions corresponding to the first few modes are approximately the same, in the $L^{2}$ norm, as that of the lake without the plate. At the same time, the ratio of the lower hydroelastic eigenfrequencies to that of the basin approaches a value that is approximately independent of the mode number $n$. It is evident that this value depends strongly on the basin depth. This type of behaviour is observed in Figs. 5 and 6 when $l_{P} / L \rightarrow 1$. In such cases, the effect of the stiffness being very minor, the basin behaves like one with a reduced depth occurring by taking into account the plate draft.

In order to examine the effect of the plate's position inside the basin, a plate of length $l_{P}=400 \mathrm{~m}$ is considered inside a pond of length $L=1000 \mathrm{~m}$ and depth $B=2 \mathrm{~m}$. The plate now is positioned such that its left edge is only $50 \mathrm{~m}$ away from the left endpoint of the basin. Figure 7, shows the first 6 eigenstates of the configuration. It is observed that significant differences in the upper surface elevation occur when comparison is made to the response of the basin without the floating body (dashed blue line). When comparisons are made with the case of the same plate located at the middle of the basin (Fig. 4), significant differences are observed as well. This fact suggests that the plate's position inside the basin affects the eigenstates and could be an important design parameter.

The effect of the plate's position is studied in Figs. 8 and 9. Again, the case of the very shallow basin $(2 \mathrm{~m})$ is considered along with a basin of $3 \mathrm{~m}$ and one of $4 \mathrm{~m}$ depth. The length of the floating plate in all cases is $l_{P}=400 \mathrm{~m}$. The distance between the basin's left endpoint and the plate's left edge is denoted by $\xi$. 

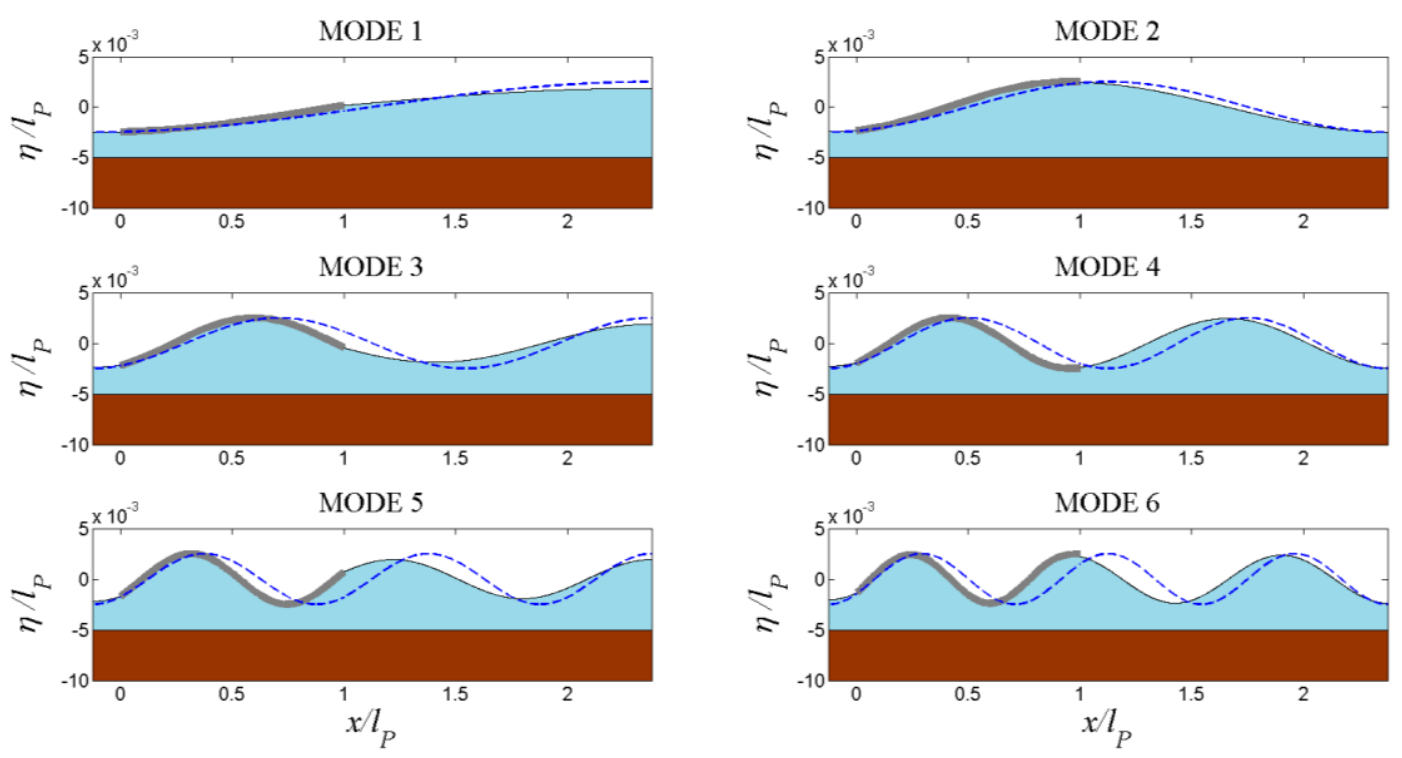

Figure 7. Eigenstates for a floating plate of span 400m inside a closed shallow basin (1000m length and $2 \mathrm{~m}$ deep). The mode of the basin without the plate is plotted as blue dashed line.

Figure 8 shows the effect of the plates position on the upper surface elevation-plate deflection as a function of the nondimensional distance $\xi / L$, for the three different basin depths. As expected, the curves are symmetric with respect to the value $\xi / L=0.3$, which corresponds to the plate's midpoint be located exactly halfway the basins length. That is, the system is indifferent whether distance $\xi / L$ is measured with respect to the left or the right endpoint of the basin. The effect of the plate's location is more intense when the plate is nearer the basin's boundary points. Furthermore, differences in the upper surface elevation, with respect to the pond without the plate, are amplified when the depth decreases and the number of the considered mode increases.

Figure 9, shows the effect of the plate's position on the eigenfrequencies. In this case, the location of the maximum intensity of the effect depends strongly on the number of the mode. In general, the effects appear to be more intense on the first eigenfrequency. The variation of the ratio $\omega_{n} / \Omega_{n}$ with respect to $\xi / L$ becomes more oscillatory as $n$ increases. At the same time, the amplitude of this oscillation reduces.

Finally, we remark the fact that in the above examples, the number of eigenfunction nodes increases with frequency like a typical Sturm-Liouville system. 

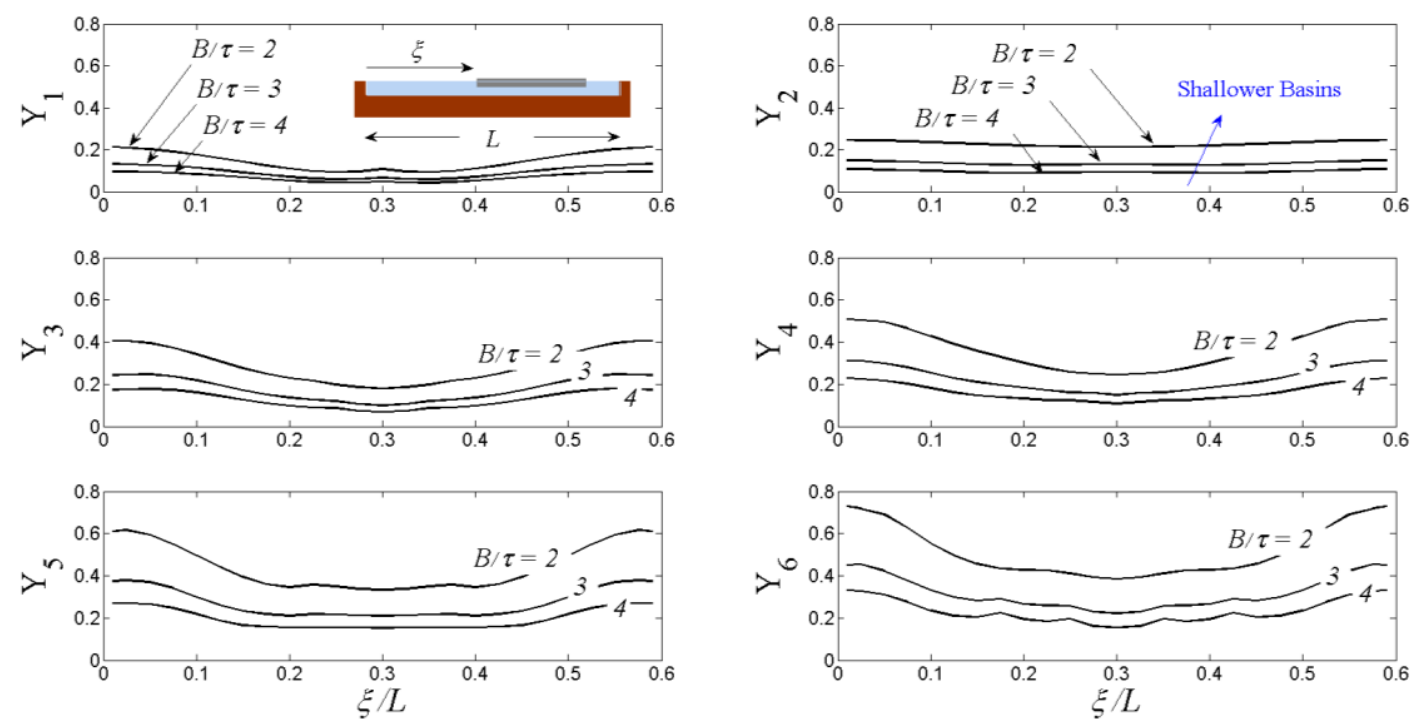

Figure 8. Effect of the floating plate's position on the upper surface elevation - plate deflection in a shallow basin of depth $B \mathrm{~m}$. The plate thickness is $\tau=1 \mathrm{~m}$.
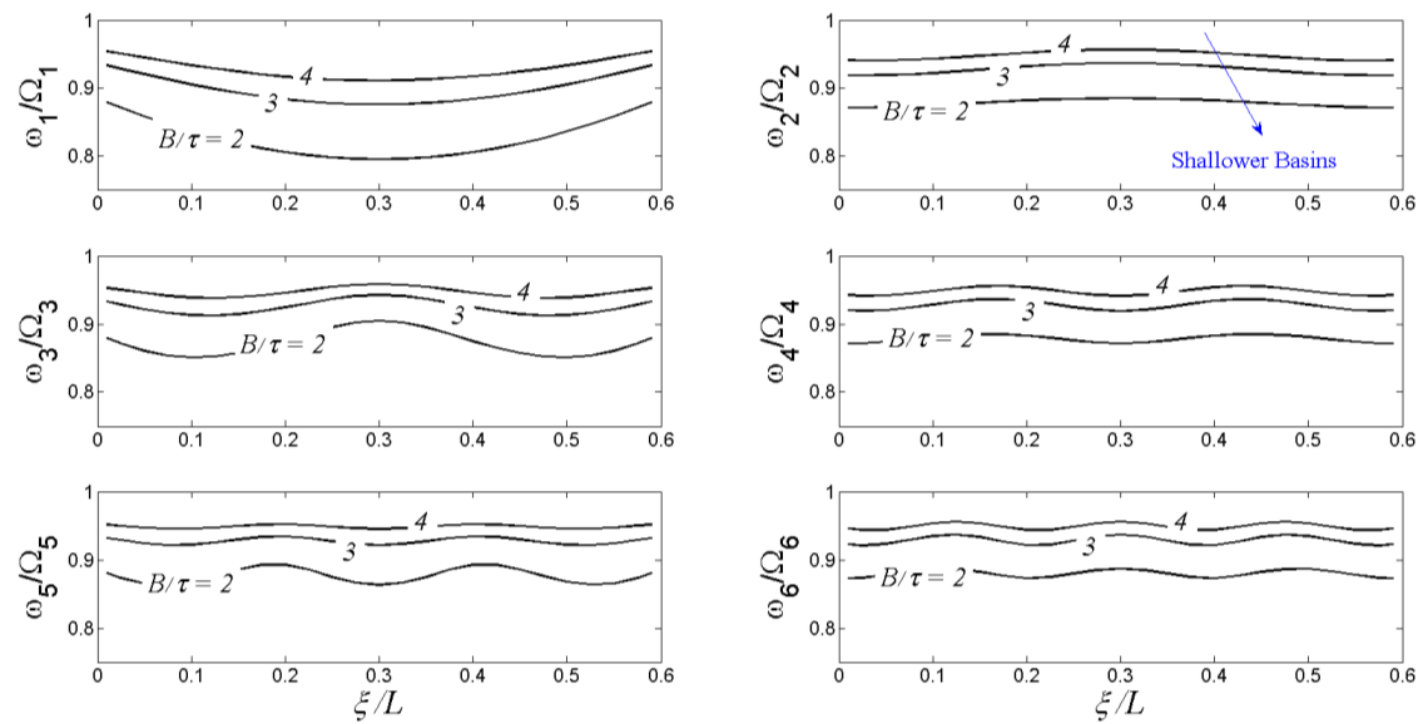

Figure 9. Effect of the floating plate's position on the eigenfrequencies of a shallow basin having depth $B \mathrm{~m}$. The plate thickness is $\tau=1 \mathrm{~m}$.

\subsection{Effect of Plate Draft, Mass and Stiffness}

The effect of the draft, mass and stiffness of the floating plate are studied in this section. A basin of length $L=1000 \mathrm{~m}$ and depth $3 \mathrm{~m}$ is considered and a plate with span $l_{P}=200 \mathrm{~m}$ is floating with its center located at the middle of the basin. The mass parameter $m$ is allowed to vary, by varying the plate density value between 0 and 950 $\mathrm{kg} / \mathrm{m}^{3}$. The plate's thickness is $\tau=1 \mathrm{~m}$. Two different values are selected for the 
Young's modulus of the floating plate material, namely $E=5 \cdot 10^{6} \mathrm{~N} / \mathrm{m}^{2}$ and $E=5 \cdot 10^{9} \mathrm{~N} / \mathrm{m}^{2}$, producing the stiffness parameters $K=1.823 \cdot 10^{-9}$ and $K=1.823 \cdot 10^{-6}$ respectively. The effect of the material parameters on the eigenfrequencies is presented in the left column plots of Fig. 10, while the effect on the eigenmodes is depicted in the right column plots of Fig. 10. Solid lines represent the variation when the plate draft is included in the model and dashed lines are used for the case where the draft is not taken into account. The circle markers are used for the curves corresponding to the more flexible elastic plate.

The increase of the plate mass reduces the eigenfrequencies and increases the differences in the upper surface elevation, with respect to upper surface elevation of the pond without the plate. However, these alterations appear to be caused only because of the related increase of the draft. If the draft is ignored the eigenstates are practically identical to those of the pond without the plate. The increase of the plate stiffness appears to have a very small influence on the eigenstates for the first few modes and the range of Young's modulus values examined. Still its effect appears to increase with increasing mode number and it is therefore expected that higher modes will be more sensitive to flexibility variations. In all cases, the plate draft is the crucial parameter, affecting more explicitly the eigenstates of the system.
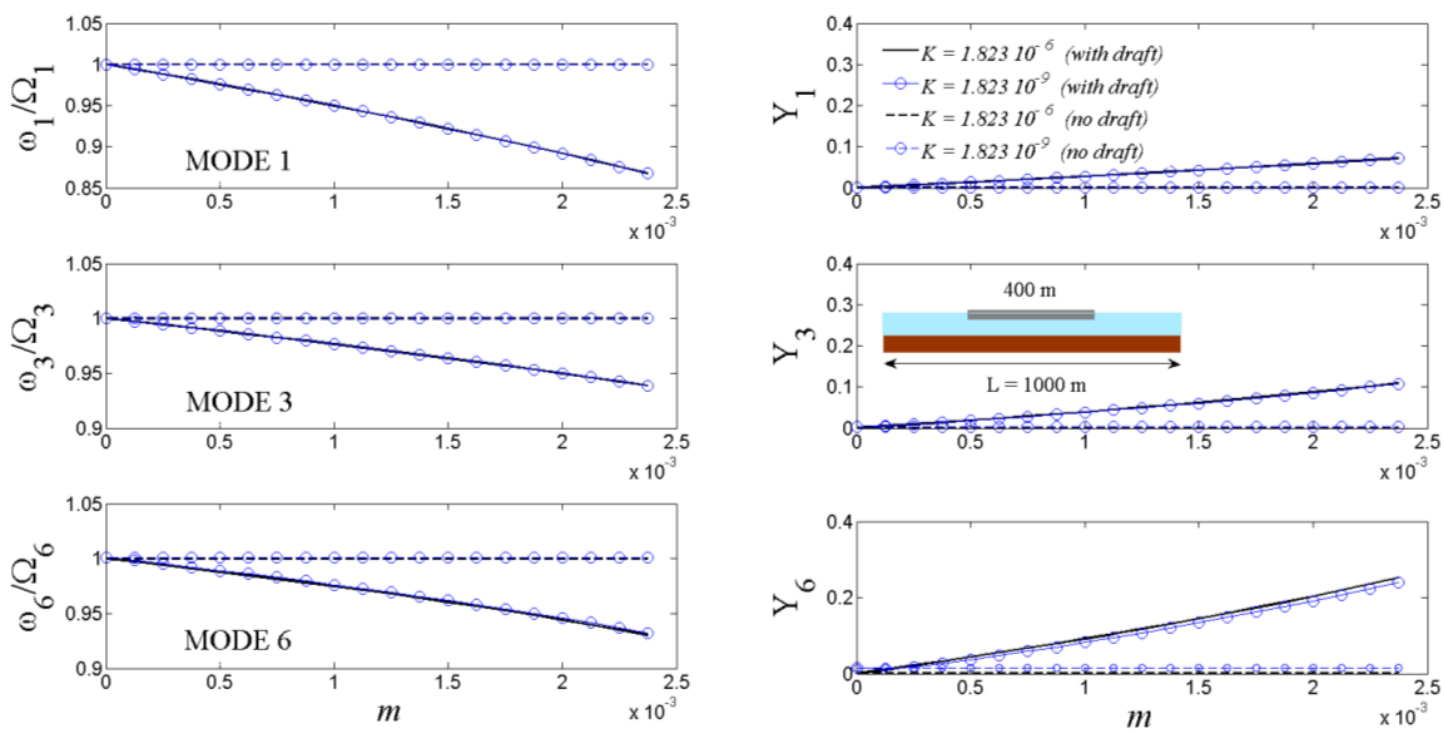

Figure 10. Effect of the floating plate's draft, mass and stiffness on the eigenfrequencies (left column) and eigenmodes (right column) of a $3 \mathrm{~m}$ deep basin.

It is interesting, although probably not relevant to the analysis of very large floating PV platforms or floating ice formations, to examine the case where the stiffness of the plate increases even further. To this end a Young's modulus value of $E=5 \cdot 10^{11} \mathrm{~N} / \mathrm{m}^{2}\left(K=1.823 \cdot 10^{-4}\right)$ is assumed while the other parameters are: $l_{P}=400 \mathrm{~m}, L=1000 \mathrm{~m}, B=3 \mathrm{~m}, v=0.3, \tau=1 \mathrm{~m}$ and $\rho / \rho_{w}=0.9$. The response of the pond is shown in figure 11 

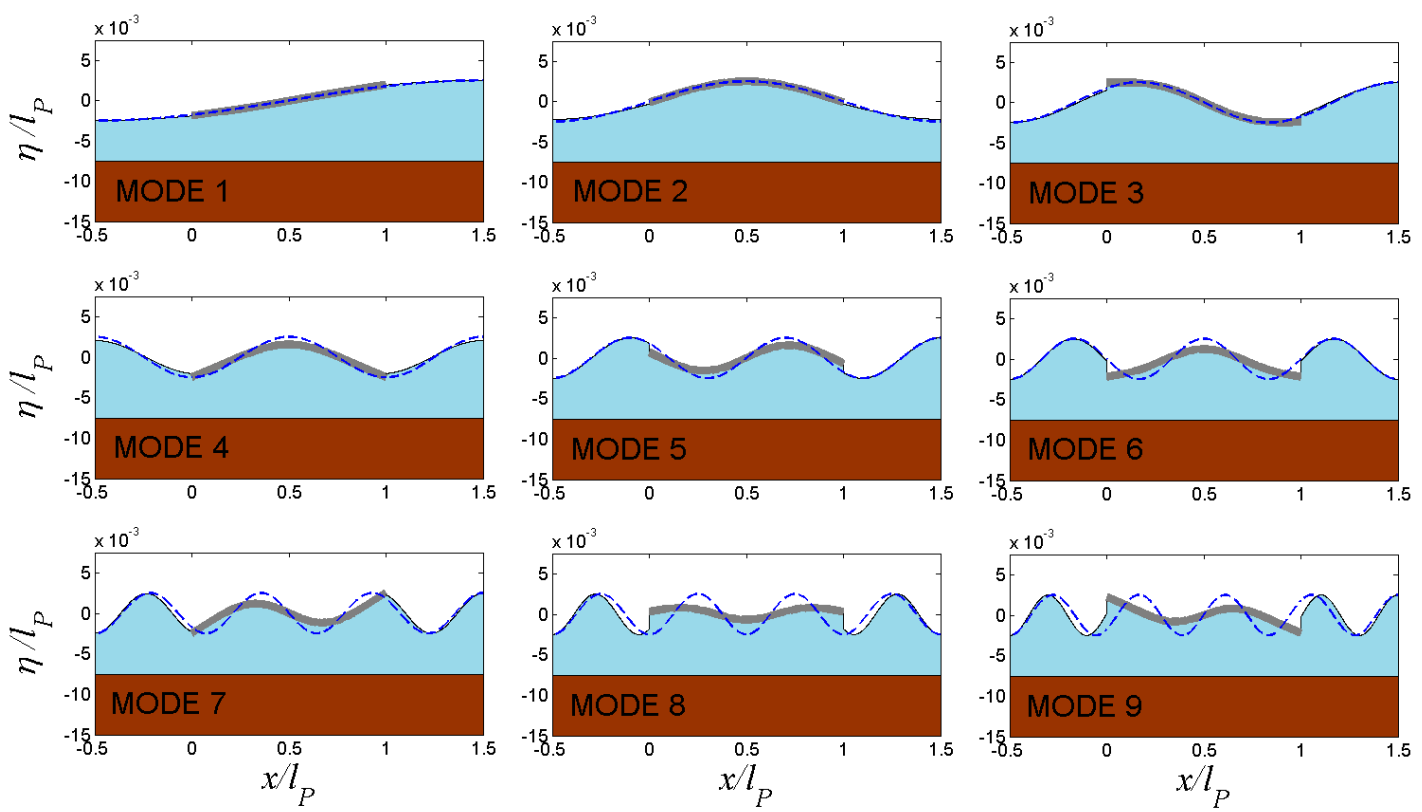

Figure 11. Eigenstates for a stiff ( $E=5 \cdot 10^{11} \mathrm{~N} / \mathrm{m}^{2}$ ) floating plate of span $400 \mathrm{~m}$ inside a closed shallow basin (1000m length and $3 \mathrm{~m}$ deep). The mode of the basin without the plate is plotted as blue dashed line.

\subsection{Multiple Floating Plates}

As a first case study a basin of $3 \mathrm{~m}$ depth and $1000 \mathrm{~m}$ length with two floating plates is considered. The first plate has a length of $400 \mathrm{~m}$ and is placed near one edge of the basin. In particular, the left boundary of the plate is positioned $10 \mathrm{~m}$ away from the left end point of the basin. The second plate has a span of $200 \mathrm{~m}$. Both plates have stiffness $E=5 \mathrm{GPa}$, Poisson's ratio $v=0.3$, density $\rho=900 \mathrm{~kg} / \mathrm{m}^{3}$ and thickness $\tau=1 \mathrm{~m}$. The control parameter is the distance between the floating plates denoted as $\delta$, while $l_{P}=400 \mathrm{~m}$. It is expected that when distance $\delta$ varies, the eigenstates of the system will be altered. The effect of the distance $\delta$ on the first four eigenstates is presented in figure 12. The indicator for the shift of the spectrum is now presented as $1-\omega_{n} / \Omega_{n}$ so that the effect on the eigenfrequencies and the effect on the eigenfunctions can be efficiently plotted in the same diagram.

It is evident that the effect of the distance $\delta$ on the eigenstates depends strongly on the specific mode examined. For the first mode (wavelength of magnitude comparable to the basin length), the effect on the eigenfrequency is more significant when the distance between the plates is small, while the effect on the eigenfunction is intensified for large values of $\delta$. This result suggests that for the first mode and for the parameters examined, when the two plates are very close to each other, the system behaves similarly to a basin with one plate having the combined length of the two. An interesting observation is that the effect of $\delta$ on the upper surface elevation, for the 
case of the first and second mode, has a minimum which roughly coincides with the $\delta$ value producing the maximum shift of the eigefrequency.

The eigenstates of the system examined are plotted in figure 13 for the case $\delta / L=0.3$. The dashed line corresponds to the upper surface elevation of the water in a pond without floating plates. It is again observed that the effect of the floating bodies on the upper surface elevation is more intense for the higher modes.
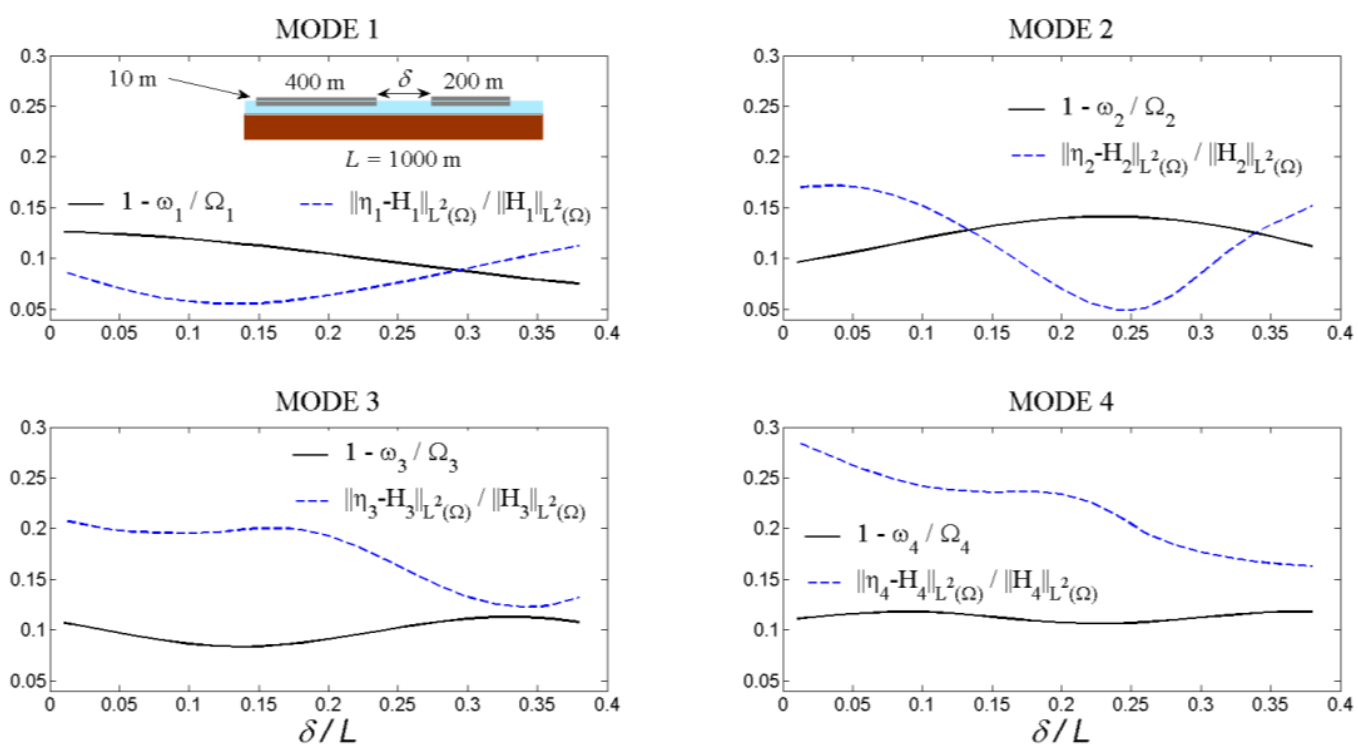

Figure 12. Effect of two floating plates on the eigenstates of a $3 \mathrm{~m}$ deep basin, as a function of the distance between the plates.
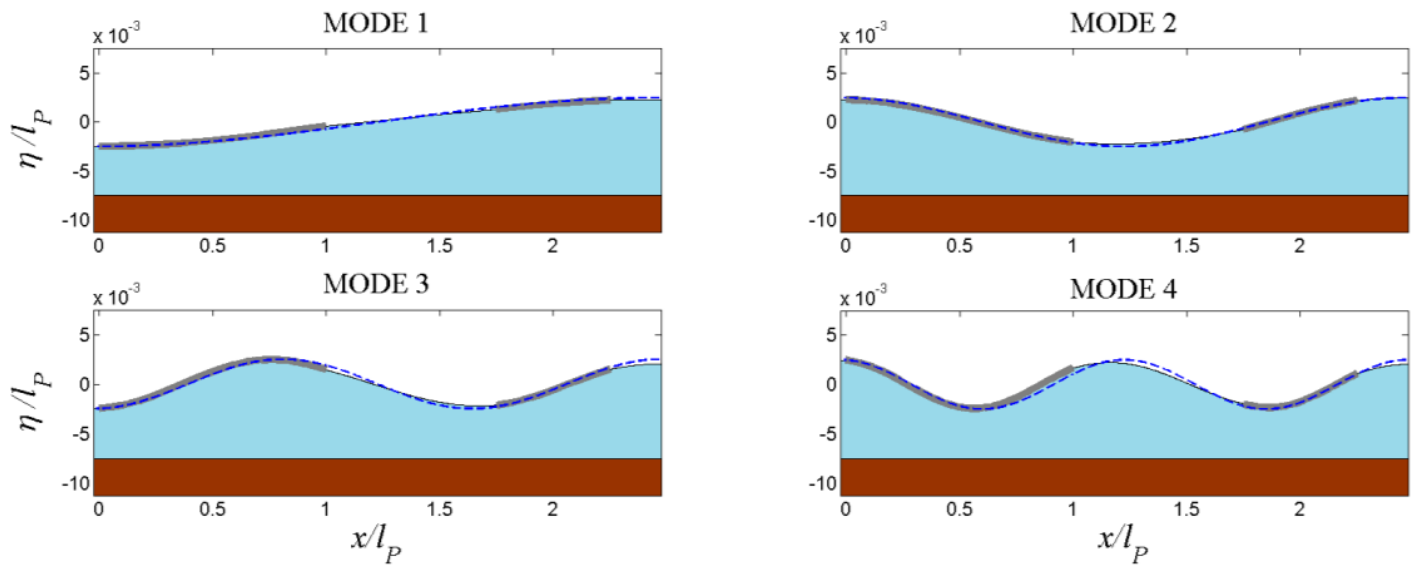

Figure 13. First four eigenstates of a $3 \mathrm{~m}$ deep basin with two floating plates, for $\delta / L=0.3$. The large plate is located $10 \mathrm{~m}$ from the basin edge. 


\subsection{Variable Bathymetry Basins}

In this section, a variable bathymetry basin will be studied. A pond with infinite width, length $L$ and a linearly varying depth profile is considered. The shallow end of the pond is assume to have depth $B_{o}$ and the slope is denoted as $\sigma$. The eigenstates of the pond with no floating plates are simulated by the equation (considering the nondimensional variables introduced in section 3)

$$
\frac{d}{d x}\left(\left[B_{o}+\sigma x\right] \frac{d \Phi}{d x}\right)+\Omega^{2} \Phi=0
$$

The same form has been used by [29] for the study of long wave propagation over piece-wise linear seabed profiles. Setting $\left[B_{o}+\sigma x\right] \frac{d \Phi}{d x}=\varphi$, differentiating eq. (6.5), and using the substitution $z=B_{o}+\sigma x$, results to

$$
\frac{d^{2} \varphi}{d z^{2}}+\left(\frac{\Omega}{\sigma}\right)^{2} z^{-1} \varphi=0
$$

The analytical solution of the above equation is (see Polyanin and Zaitsev [30])

$$
\varphi(z)=\sqrt{z}\left[C_{1} J_{1}\left(2 \Omega \sigma^{-1} \sqrt{z}\right)+C_{2} Y_{1}\left(2 \Omega \sigma^{-1} \sqrt{z}\right)\right] .
$$

Applying the zero velocity boundary conditions $\varphi(0)=\varphi\left(L l_{P}^{-1}\right)=0$ nontrivial solutions are obtained, setting $\beta=2 \Omega \sigma^{-1} \sqrt{B_{o}}$, when

$$
\mathrm{F}(\Omega)=J_{1}(\beta) Y_{1}\left(\beta \sqrt{1+\sigma L /\left(B_{o} l_{p}\right)}\right)-Y_{1}(\beta) J_{1}\left(\beta \sqrt{1+\sigma L /\left(B_{o} l_{p}\right)}\right)=0,
$$

Approximate formulas for the roots of $\mathrm{F}(\Omega)$, in terms of the quantity $1+\sigma L /\left(B_{o} l_{p}\right)$ can be found in [31], page 347, section 9.5.27. For the specific case study two basins are considered, one with $\sigma=0.004$ and one with $\sigma=0.009$. For both basins it is $L=1000 \mathrm{~m}$ and $B_{o}=1 \mathrm{~m}$. The first six roots of the transcendental equation (6.6) for both basins have been calculated using Newton-Raphson iterations and initial values based on the formulas in [31].

The effect of a floating plate with span $l_{P}=300 \mathrm{~m}$, stiffness $E=5 \mathrm{GPa}$, Poisson's ratio $v=0.3$, density $\rho=900 \mathrm{~kg} / \mathrm{m}^{3}$ and thickness is $\tau=1 \mathrm{~m}$ on the first six eigenfrequencies is plotted in Fig. 14 as a function of the plate location. Similarly to Figs. 8 and $9, \xi$ denotes the distance between the left lateral boundary of the plate and the left end point of the basin. For both basins considered, the effect intensifies when the plate floats at the shallow region of the basin. Furthermore, the spectrum is altered to a greater extent when the mean depth is smaller. 
Subsequently, for the case $\sigma=0.004$ a floating plate of $l_{P}=300 \mathrm{~m}$ is assumed to be located at distance $50 \mathrm{~m}$ form the shallow end. The floating plate has the same properties as above. The first six eigenstates of the system are depicted in Fig. 15. The presence of the floating plate alters significantly the response of the system, in terms of the eigenmodes.
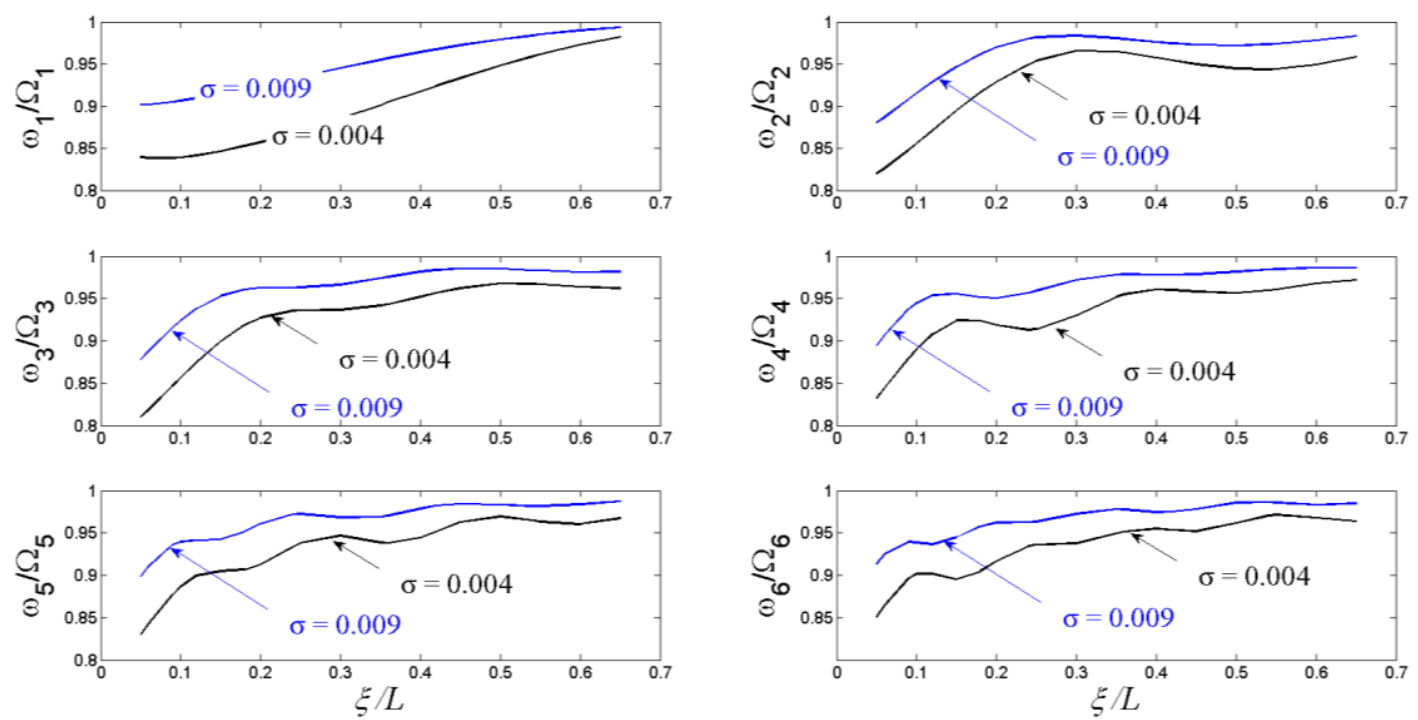

Figure 14. Effect of a floating plate's position on the eigenfrequencies of two basins with constant, mild slope. The distance of the plate from the basin shallow edge is denoted by $\xi$.
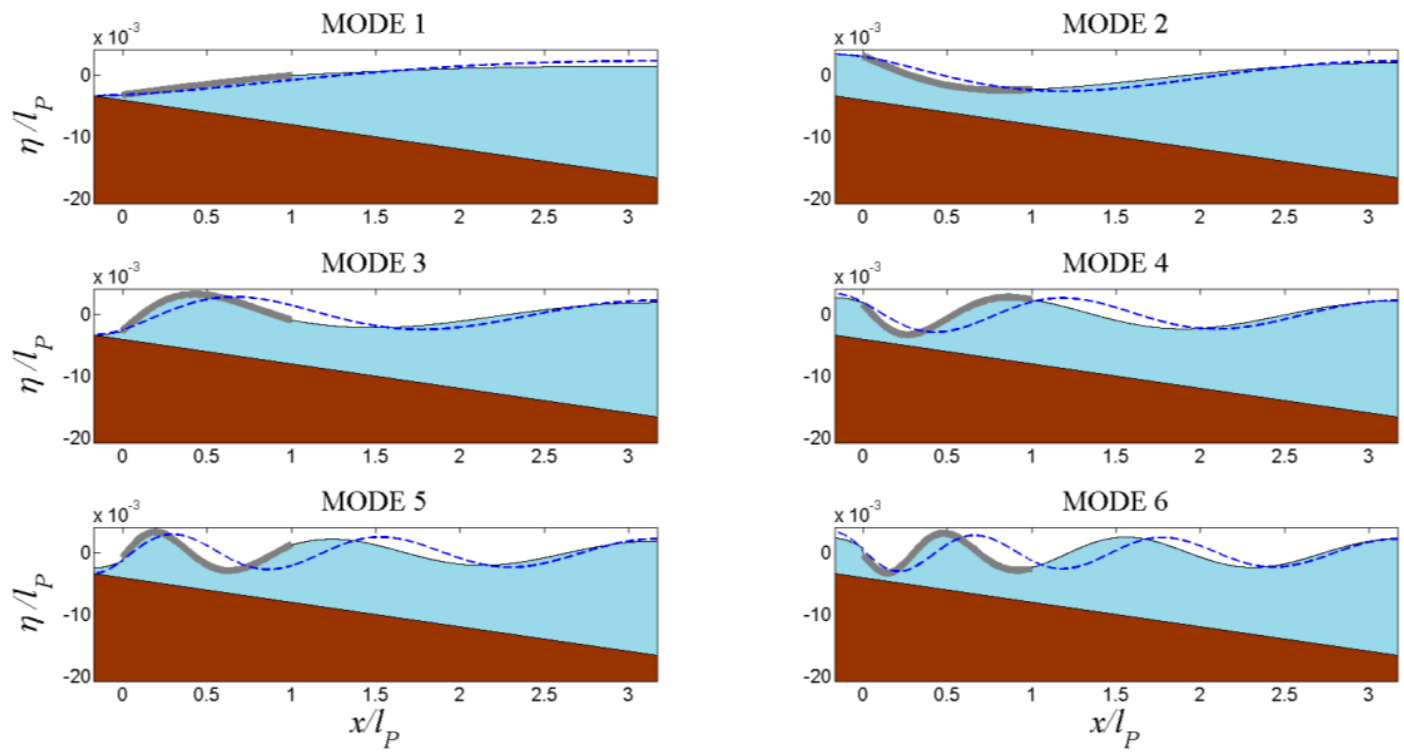

Figure 15. First six eigenstates of a $1000 \mathrm{~m}$ long, deepening pond with $\sigma=0.004$ and a floating plate of span $300 \mathrm{~m}$ located $50 \mathrm{~m}$ away from the shallow end. 


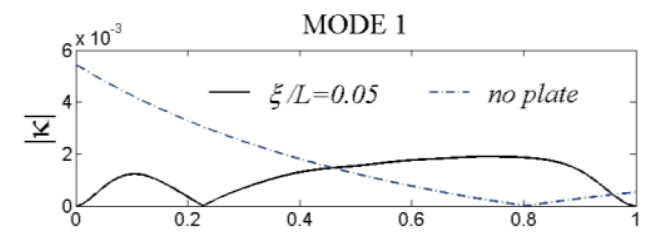

MODE 3

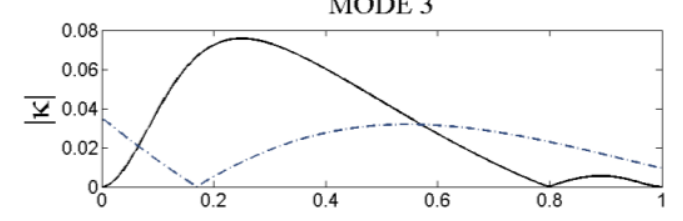

MODE 5

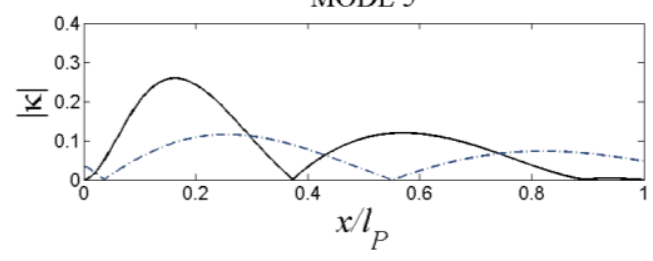

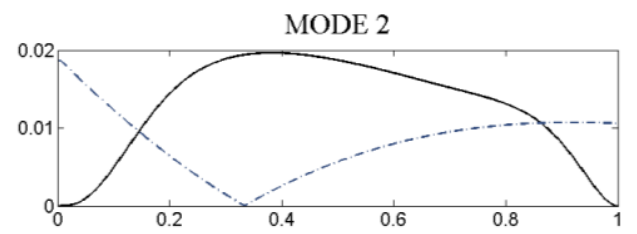

MODE 4

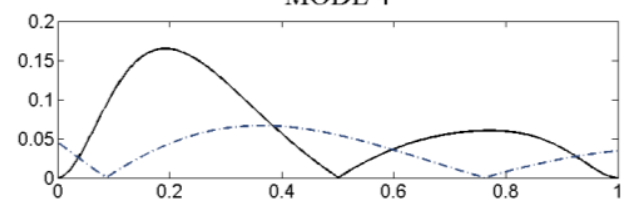

MODE 6

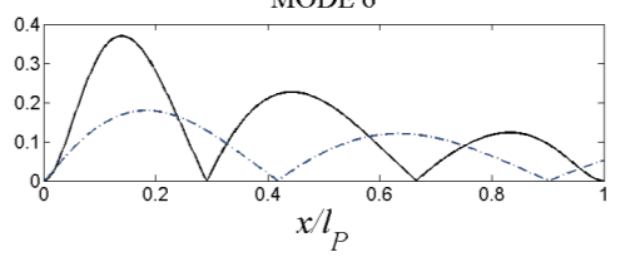

Figure 16. Curvature of the plate at the eigenstates presented in Fig. 14 (solid line) and curvature of the basin's upper surface elevation at the same span when no plate is present (dashed).

The absolute value of the curvature along the span of the beam is plotted in Fig. 16. The curvature is an indicator of the normal stress magnitude inside the beam, generated due to the induced bending moments. For small deflections, the curvature $\kappa$ is the second spatial derivative of the deflection $\eta$ and the bending moment is proportional to the curvature $\mathrm{M}_{b} \sim \kappa=\eta_{x x}$. The curvature of the free surface elevation without a floating plate is plotted as well (using a dashed line) for comparison purposes. It is observed that the peak curvature value increases as the mode number increases. Apart from the case of the first mode, the maximum curvature in the presence of the floating plate is significantly higher. The proposed finite element procedure is an efficient means to calculate bending moment distributions, and consequently stresses, along the floating plates. In situations where the depth is small compared to the thickness of the plate, e.g. in the above configuration, 3D effects in the response of the system are expected to become significant. In such cases the analysis should be supplemented with the use of more elaborate models that can take into account through the depth variations of the hydorelastic field and possible local singularities near the corner boundaries of the fluid-floating structure system [32].

The same basin and floating plate are studied in the following example, with the plate now being located at the middle of the pond $(\xi=350 \mathrm{~m})$. The first six eigenmodes of the system, depicted in Fig. 17, indicate that the differences in the upper surface elevation of the plate are in this case much smaller. It might be temping thus to analyse the response of the system in terms of the pond with no floating body. However, the curvature distributions along the plate (depicted in Fig. 18), indicate differences of approximately $40 \%$, in certain cases. This example reveals the significance of the coupled hydroelastic analysis, even in situations where the 
differences between the upper surface eigenmodes appear to be negligible. In the case where the stiffness is small and at the same time the draft of the plate, compared to the depth of the water, is small, the effect of the floating plate is insignificant in the sense that the eigenfrequencies of the basin and the $\mathrm{L}^{2}$ norm of the eigenfunctions are virtually not altered. In such cases the problem can be treated as a singularly perturbed problem of the basin eigenstates. Finally, it is mentioned that the model presented can be used for the treatment of floating plates rigidly connected to the basin banks, as well.
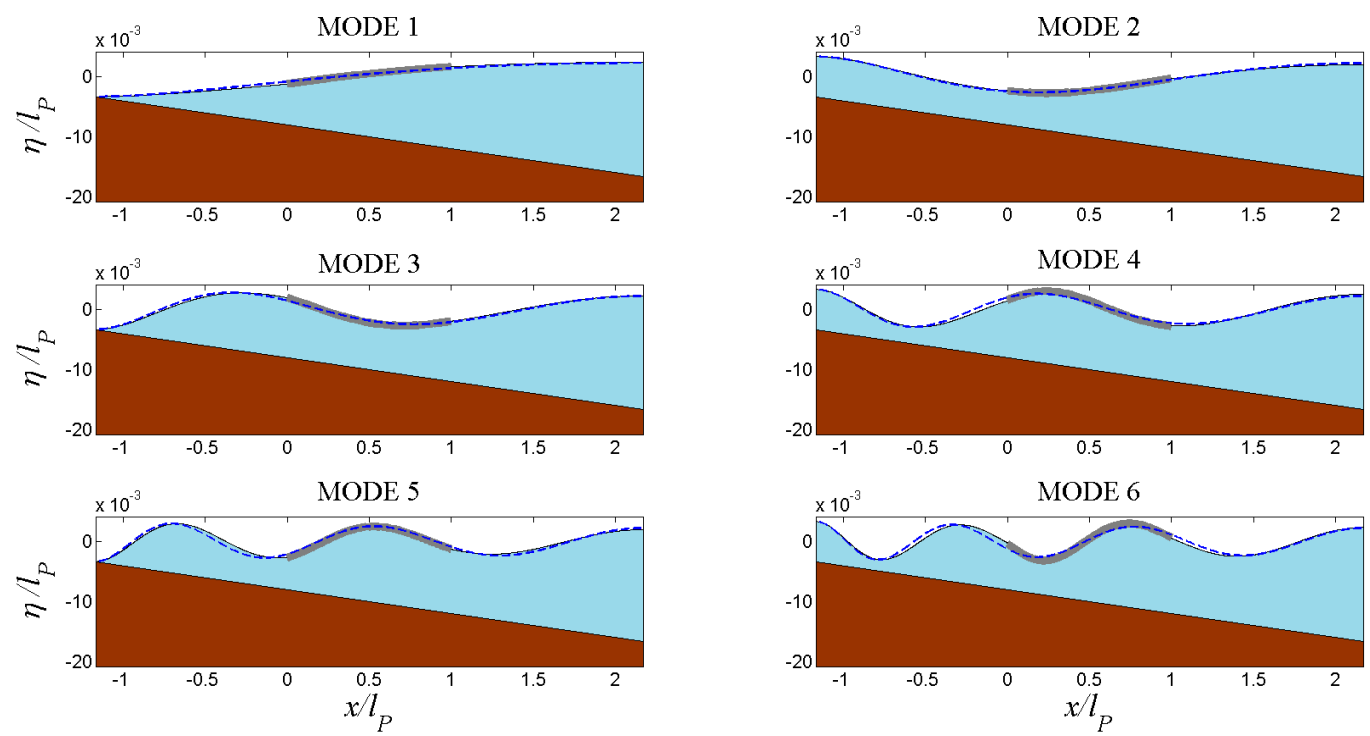

Figure 17. First six eigenstates of a $1000 \mathrm{~m}$ long, deepening pond with $\sigma=0.004$ and a floating plate of span $300 \mathrm{~m}$ located $350 \mathrm{~m}$ away from the shallow end.

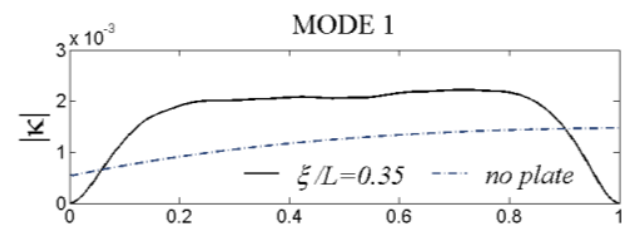

MODE 3

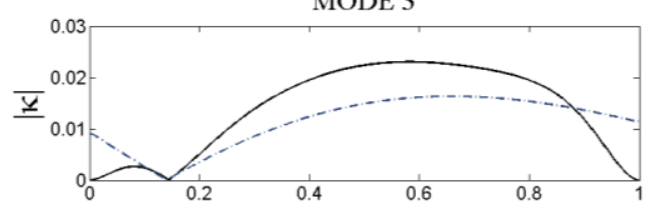

MODE 5

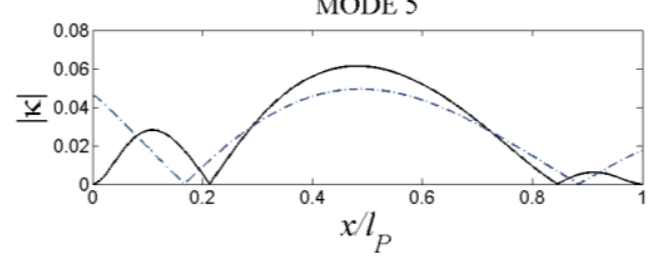

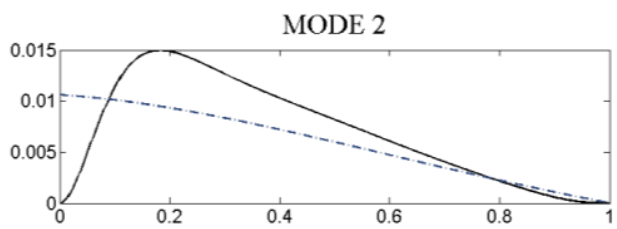

MODE 4

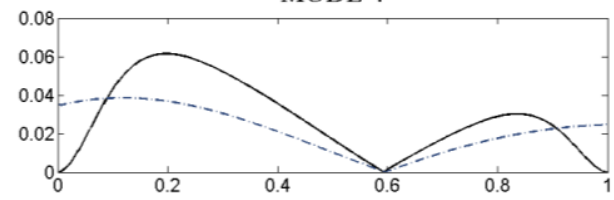

MODE 6

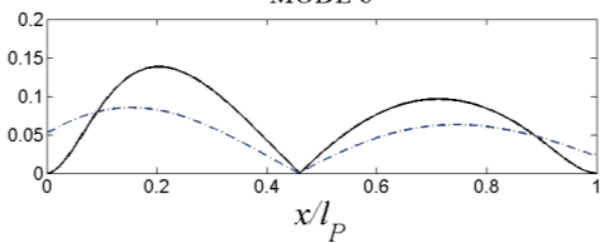

Figure 18. Curvature of the plate at the eigenstates presented in Fig. 16 (solid line) and curvature of the basin's upper surface elevation at the same span when no plate is present (dashed line). 


\section{Conclusions and Future Developments}

The hydroelastic response of closed shallow basins with floating slender plates of large span has been studied, based on the variational form of a simplified model, and special hydroelastic finite elements. The phenomenon is found to be governed by a quadratic eigenvalue problem for the eigenfrequencies and eigenmodes of the basinfloating body system. Several benchmark examples are presented for certain simplified configurations in order to study the effects of parameters like the plate's span, position, draft, mass, stiffness and depth of the basin. The presence of the plate has been found to alter significantly the eigenstates of the pond if the depth of the basin is relatively small compared to the plate draft. The plate draft is a crucial parameter for the problem. When the draft is significant, the plate span and position inside the basin play a decisive role. The stiffness of the floating plate is shown to have only minor effects for the values of Young's modulus examined, and influences mostly higher modes. The hydroelastic analysis is important even in cases where the depth, compared to the draft, increases and the upper surface elevation differences, in the presence or absence of the floating plate, appear to be insignificant. This is due to the underestimation in the maximum curvature, and hence bending moment values, that can occur if the upper surface elevation of the system is calculated without the plate. Finally, the finite element method appears to be an efficient, powerful and versatile tool for hydroelastic simulations of this type and particular involved problems like cases of variable bathymetry basins or when multiple floating plates are considered. To study more realistic geometries and floating plates of finite width, finite elements in two horizontal dimensions are needed [33]. Future work is planned towards the development of such a model, based on the variational form presented in sections 3 and 5. A 2D Finite Element method of this kind can be used for the simulation of multiple floating plates of irregular shape, incorporating the effects of variable bathymetry, variable plate properties and simple anchoring configurations [34]. It is envisioned that this new methodology will constitute an efficient tool for the analysis of demanding problems of VLFS hydroelasticity [35] like the hydroelastic resonant behaviour of closed basins.

Competing interests: the authors verify that they have no competing interests with other parties

Funding statement: this research was not funded by any funding bodies

\section{References}

1. Jackson JR. 1833 On the seiches of lakes, Journal of the Royal Geographical society of London, 3, 271-275. 
2. Kvale A. 1955 Seismic seiches in Norway and England during the Assam earthquake of August 15, 1950, Bull. Seismol. Soc. Am., 45(2), 93-113.

3. McGarr A. and Vorhis RC. 1968, Seismic seiches from the March 1964 Alaska earthquake, U.S. Geol. Surv. Prof. Pap., 544, E1-E43.

4. Forel FA. 1892/1895/1904, Le Léman, Monographie Limnologique, (3 Volumes), Librairie de l'Université, Lausanne

5. Chrystal G.1906 On the hydrodynamical theory of seiches, T. Roy. Soc. Edin.Earth, 41, 599-649.

6. Farhadzadeh A. 2017, A study of Lake Erie seiche and low frequency water level fluctuations in the presence of surface ice, Ocean Engineering 135, 117-136

7. McGarr A. 1965, Excitation of seiches in channels by seismic waves, J. Geophys. Res., 70(4), 847-854.

8. Rabinovich AB. 2009, Seiches and harbour oscillations, in: Handbook of Coastal and Ocean Engineering (Ed. Young CK), World Scientific, Singapore

9. Robertson J. et al. 1755 An extraordinary and surprising agitation of the waters, though without any perceptible motion of the earth, having been observed in various parts of this Island, both maritime and inland, on the same day, and chiefly about the time, that the more violent commotions of both earth and waters so extensively affected many very distant parts of the globe; The following accounts, relating to the former, have been transmitted to the Society; in which are specified the times and places when and where they happened." Philosophical Transactions (1683-1775), 49, 351-398.

10. Bondevik S, Gjevik B, Mathilde B. Sørensen MB 2013, Norwegian seiches from the giant 2011 Tohoku earthquake, Geophysical Research Letters, 40, 3374-3378

11. Palmer MD, Izatt JB, 1972 Lake movement with partial ice cover, Limnol. Oceano., 17, 403-409.

12. Sturova IV. 2007. Effect of ice cover on oscillations of fluid in a closed basin, Izvestiya, Atmospheric and Oceanic Physics, 43, 1, 112-118

13. Trapani K., Santafé MR. 2015 A review of floating photovoltaic installations: 2007-2013, Prog. Photovolt. 23(4), 524-532

14. https://www.theguardian.com/environment/2016/feb/29/worlds-biggest floatingsolar-farm-power-up-outside-london

15. Bromirski, P.D., Diez, A., Gerstoft, P., Stephen, R.A., Bolmer, T., Wiens, D.A., Aster, R., Nyblade, N., 2015. Ross ice shelf vibrations. Geophys. Res. Lett. 42. 7589-7597

16. Squire, V.A., Robinson W. H., Meylan M., Haskell, T.G. 1994, Observations of flexural waves on the Erebus-ice-tongue, Mcmurdo-sound, Antarctica, and nearby sea-ice', Journal of Glaciology, 40, 377-385

17. Godin, O. A \& Zabotin, N. A. (2016). Resonance vibrations of the Ross Ice Shelf and observations of persistent atmospheric waves. Journal of Geophysical Research: Space Physics, 121, 10157-10171

18. Sergienko, O. V. (2013). Normal modes of a coupled ice-shelf/sub-ice-shelf cavity system. Journal of Glaciology, 59 (213), 76-80. 
19. Meylan MH, Bennetts LG, Hosking RJ, Catt E. 2017 On the calculation of normal modes of a coupled ice-shelf/sub-ice-shelf cavity system, J. Glaciol., 63 751-754

20. Papathanasiou, T. K., Karperaki, A. E., Theotokoglou, E. E., \& Belibassakis, K. A. 2015. Hydroelastic analysis of ice shelves under long wave excitation. Natural Hazards and Earth System Sciences, 15(8), 1851-1857.

21. Muhammad I., Meylan MH, Lamichhane B, Bennetts LG 2018 Time-domain and modal response of ice shelves to wave forcing using the finite element method, $J$. Fluid Struct. 80, 113-131.

22. Stoker JJ. 1957 Water Waves: The mathematical Theory with Applications, New York: Wiley-Interscience.

23. Timoshenko S. and Woinowsky-Krieger S. 1959 Theory of plates and shells, McGraw Hill, New York

24. Ciarlet PG. 2002, The finite element method for elliptic problems, SIAM classics in Applied Mathematics.

25. Tisseur F, Meerbergen K. 2001, The quadratic eigenvalue problem, Siam Review; 43, 2, 235-286

26. Papathanasiou TK, Karperaki A, Theotokoglou EE, Belibassakis KA. 2014 A higher order FEM for time-domain hydroelastic analysis of large floating bodies in an inhomogeneous shallow water environment. Proc $R$ Soc A;471:20140643.

27. Lancaster P 1991. Quadratic eigenvalue problems, Linear Algebra Appl., 150; 499-506.

28. Squire VA. 2008 Synergies Between VLFS Hydroelasticity and Sea Ice Reasearch. Int. J. Offshore Polar Eng. 18, 1-13.

29. Kanoğlu U, Synolakis CE 1998 Long wave runup on piecewise linear topographies, J. Fluid Mech., 374, 1-28

30. Polyanin AD, Zaitsev VF. 2003. Handbook of exact solutions for ordinary differential equations, $2^{\text {nd }}$ Edition, Chapman \& Hall/CRC.

31. Abramowitz M, Stegun IA (Eds.) 1972. Handbook of mathematical functions, United States Department of Commerce, National Bureau of Standards, Applied Mathematics Series 55, $10^{\text {th }}$ printing.

32. Bennets LG, Williams TD, 2010 Wave scattering by ice floes and polynyas of arbitrary shape, J. Fluid Mech., 662, 5-35

33. Wang CD, Meylan MH, 2004 A higher-order-coupled boundary element and finite element method for the wave forcing of a floating elastic plate, J. Fluid Struct. 19, 557-572

34. Karperaki AE, Belibassakis KA and Papathanasiou TK 2016, Time-domain, shallow-water hydroelastic analysis of VLFS elastically connected to the seabed, Mar. Struct., 48, 33-51

35. Korobkin A, Părău EI and Vanden-Broeck JM, 2011 The mathematical challenges and modelling of hydroelasticity Phil. Trans. R. Soc. A 369, 28032812 